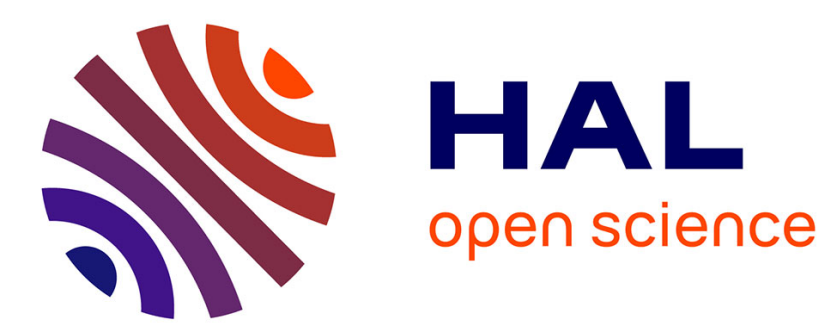

\title{
Comparison of linear solvers for equilibrium geochemistry computations
}

Hela Machat, Jérôme Carrayrou

\section{To cite this version:}

Hela Machat, Jérôme Carrayrou. Comparison of linear solvers for equilibrium geochemistry computations. Computational Geosciences, 2017, 21 (1), pp.131-150. 10.1007/s10596-016-9600-5 . hal03321650

\section{HAL Id: hal-03321650 \\ https://hal.science/hal-03321650}

Submitted on 17 Aug 2021

HAL is a multi-disciplinary open access archive for the deposit and dissemination of scientific research documents, whether they are published or not. The documents may come from teaching and research institutions in France or abroad, or from public or private research centers.
L'archive ouverte pluridisciplinaire HAL, est destinée au dépôt et à la diffusion de documents scientifiques de niveau recherche, publiés ou non, émanant des établissements d'enseignement et de recherche français ou étrangers, des laboratoires publics ou privés. 


\title{
2 Comparison of linear solvers for equilibrium geochemistry 3 computations
}

\author{
4 Hela Machat $^{1,2} \cdot$ Jérôme Carrayrou ${ }^{1}$
}

Received: 11 February 2015 / Accepted: 24 October 2016

C) Springer International Publishing Switzerland 2016 to address this issue. One of these tools, reactive trans- 28 port models, was first limited to laboratory experiments 29and was then extended to field problem comprehension. In 30 recent decades, reactive transport models have increased in 31 complexity and efficiency, and they are now used in many 32 fields. Reactive transport models have been used to study 33 the transport of contaminants, such as heavy metals $[1,2] 34$ and radioelements [3-5]. Because of the increasing inter- 35 est in questions related to climate change, many studies 360 n reactive transport have been conducted to examine the 37 possibility of geologic $\mathrm{CO}_{2}$ sequestration [6-10].

Under the wide variety of models and cases lies a common mathematical description [11-13]. Transport is usually 40 described by an advection-dispersion equation, and the 41 chemistry is formulated under thermodynamic equilibrium. 42 A widely used approach to solve these reactive transport 43 problems is the operator splitting approach [14]. Using this 44 approach, the transport and chemical operators are solved 45 separately at each time step and iteratively for some for- 46 mulations. As a consequence, the chemistry operator has to 47 be solved at least once per mesh cell per time step. This 48 is one reason for the high computational cost of reactive 49 transport modelling. Some authors have reported that 8050 to $90 \%$ of the computation time is dedicated to chem- 51 ical computation. Many studies have been conducted to 52 reduce the computation time required by reactive transport 53 modelling [15]. Some works have explored paralleliza- 54 tion [16], while others have focused on the methods used 55 to solve the transport operator. Nevertheless, improving 56 the resolution of the chemistry operator has been iden- 57 tified as a key point. Some authors have attempted to 58 improve the classic Newton-Raphson method [17], while 59 others have tested other methods, such as Newton-Krylov 60 $[16,18]$. 
In this work, we focus on a specific element of the problem, improving the resolution of the linearized system provided by the Newton-Raphson method. Looking to numerical methods to solve linear systems is not currently a common practice. Indeed, these methods are actually well known [19-23], and all mathematical packages for scientific computation propose several routines for this task. The motivation of this work comes from the specificity of linear systems that have to be solved for equilibrium chemistry computations. Classic tests for the resolution of linear systems [24-30] are performed using systems provided by finite element or finite volume discretization, leading to matrices that are large (at least 10,000 unknowns) and sparse. Moreover, even when ill-conditioned systems have been studied [25, 30, 31], the conditioning of the matrix coming from the chemical system is specific, as underlined by Hoffmann et al. [32]. For example, Soleymani [33] worked with an ill-conditioned system constructed from 10 $\times 10$ to $20 \times 20$ Hilbert matrices. The condition numbers then range from $3.5 \times 10^{13}$ to $6.2 \times 10^{28}$. In this work, we present

chemical tests leading to a $7 \times 7$ matrix with a condition number of approximately $10^{180}$.

We expect to find a method to increase the efficiency of a speciation or reactive transport code. Several properties are required for such a method:

(i) This method should be fast, as the linear system will be solved very often. In the case of reactive transport modelling, the system will be solved at least once per mesh cell per time step.

(ii) The method should be very robust. It should be able to solve the linear system even if it is very poorly conditioned. Because the resolution of the linear system is only part of an iterative Newton step, an accurate solution is not absolutely needed. Thus, some advanced codes (e.g. Linear Algebra Package (LAPACK) routine) that check the accuracy of the solution and return an error flag instead of an inaccurate solution are, in this work, less robust than the more rustic routines.

(iii) The method should be able to detect failure and return an error flag to the main program so that a recovery procedure can be initiated. In the case of reac- tive transport modelling, this procedure could involve rejecting the current time step and recomputing with a smaller one.

(iv) In the initial analysis, the precision of the method is not the key point. Because the linear system resolution is only a part of the Newton-Raphson iterative procedure, reasonable error is acceptable for the linear system inversion. If this error is too large, it will slow the convergence speed for the Newton-Raphson method and decrease the efficiency of the reactive transport code. In this work, errors are estimated by comparing the calculated solution to a reference solution.

Because we utilize a markedly small matrix, we did not test parallelization. All the computations were performed on a PC running Windows with 64-bit Fortran 95. Real variables are defined as double-precision real. We prefer doubleprecision computations because all the chemical codes are, to the best of our knowledge, written as double-precision real and because quadruple-precision computation is much more time consuming. Nevertheless, we have tested one method using quadruple-precision real to determine whether this development could be useful. Reference solutions are also computed using quadruple precision.

We first present the formulation of the equations describing equilibrium reactions and how they are solved using the Newton-Raphson method. This point defines the Jacobian linear system, which is the object of this work. A second part is devoted to the presentation of the chemical tests and

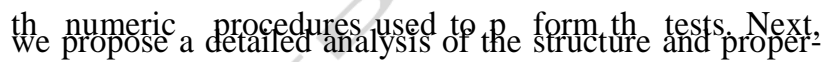

discussed. Based on this analysis, we propose an algorithm 136 to optimize the chemical computation in terms of robust- 137 ness, accuracy, and efficiency. This algorithm is evaluated 138 on the most selective test. By expanding the limits of the 139 currently used methods, we believe that our new algorithm 140 will contribute to enlarging the field of application of reac- 141 tive transport modelling. As a conclusion, we underline the 142 main advances of this work, the new perspectives and the 143 remaining obstacles.

\section{Material and methods}

\subsection{Geochemical modelling}

One efficient formulation for the computation of thermodynamic equilibrium is based on the tableau concept, referred to as Morel's table $[34,35] . N_{X}$ components $\left(X_{j}\right)$ are chosen from the $N_{c}$ species $\left(C_{i}\right)$ and are used to write the formation of each species as a combination of the components. The mass action law for the formation of the $C_{i}$ species is written with the equilibrium constant $\left(K_{i}\right)$ and the stoichiometric coefficients $\left(a_{i, k}\right)$ for each component $\left(X_{k}\right)$

$$
\left\{C_{i}\right\}=K_{i} \stackrel{N \underline{N}}{k=1}\left\{X_{k}\right\}^{a i, k}
$$

where $\left\{C_{i}\right\}$ and $\left\{X_{k}\right\}$ are the activities of species $C_{i}$ and component $X_{k}$, respectively. In this work, we define $X_{j}$ as a subset of $C_{i}$; then, $N_{x}$ is $N_{c}$ minus the number of reactions. 
If $N_{C P}$-precipitated species $\left(C p_{i}\right)$ are taken into account, the mass action law for the precipitation of $C p_{i}$ is written with the precipitation constant $\left(K p_{i}\right)$ and the stoichiometric coefficients $\left(a p_{i, k}\right)$. The saturation index $\left(\mathrm{SI}_{i}\right)$ of $C p_{i}$ is equal to its activity, which is unity for a pure solid phase

$$
\mathrm{SI}_{i}=K p_{i} \stackrel{N \underline{x}}{k=1}\left\{X_{k}\right\}^{a p_{i, k}}=1
$$

The conservation of the total concentration $\left[T_{j}\right]$ of the $\boldsymbol{j}$ th

component in the system is then written as

$$
T_{j}={ }_{i=1}^{N_{C}} a_{i, j} \cdot\left[C_{i}\right]+{ }_{i=1}^{N_{C P}} a p_{i, j} \cdot\left[C p_{i}\right]
$$

where $\left[C_{i}\right]$ is the concentration of species $\left(C_{i}\right)$ and $\left[C p_{i}\right]$ is the amount of precipitated species $\left(C p_{i}\right)$ per liquid volume unit.

A classic algorithm [17, 36-41] to describe mineral precipitation or dissolution makes an a priori hypothesis about the existence or non-existence of minerals. In this work, we assume that this hypothesis is proposed. The relationships between the activity and concentration are given by activity coefficients $\left(\gamma_{i}\right)$ calculated using specific models (Davies,

Debye-Hückel, etc.)

$\left\{C_{i}\right\}=\gamma_{i}\left[C_{i}\right]$ and $\left.x_{j}\right\}=\gamma_{j} X_{j}$

By substituting the mass action law (1) into the mass conser- 175 vation equation (3), the following relationship, which only 176 depends on the components and the precipitated species 177 concentrations, is obtained:

$$
T_{j}=\underbrace{N_{C}}_{i=1} a_{i, j} \cdot \underline{K}_{i}^{N_{X}} \cdot{ }_{k=1}^{N_{C P}}\left(\gamma_{k}\left[X_{k}\right]\right)^{a i, k}+\underbrace{}_{i=1} a p_{i, j} \cdot\left[C p_{i}\right]
$$

\section{$+$}

Combining Eqs. 2 and 5 leads to a set of $\left(N_{x} N_{C P}\right)$ non- 179 linear algebraic equations, which can be numerically solved 180through iterative methods. The concentrations of component $181\left[X_{k}\right]$ and precipitated species $\left[C p_{i}\right]$ at equilibrium are then 182 determined when the $\left(N_{X} \quad N_{C P}\right)$ objective functions $\left(Y_{j}\right) \quad 183$ are zero184

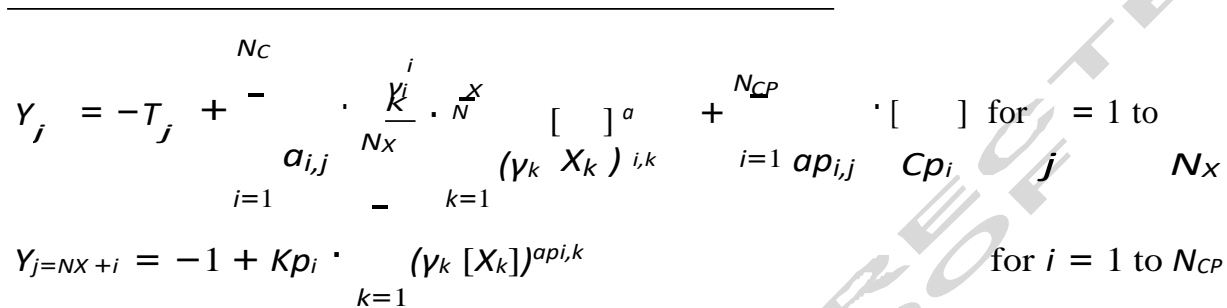

185 Using this method, it is possible to include many chemi186 cal phenomena, including activity corrections, sorption on a 187 surface using different means (such as ion exchange or sur- face complexation), and dissolution of gaseous compounds.
According to the criteria typically used for this method [17, 34, 40, 42], the convergence of the Newton-Raphson method is not checked with respect to the norm of the objective function $\|Y\|$, but the relative error defined as
199
The value of the convergence criterion $\left(\varepsilon_{N-R}=10^{-12}\right)$ is

$$
\cdot T_{j} \cdot+a_{i, j}\left[C_{i}\right] \cdot
$$$$
j=1, N x
$$
set according to usual practice.

\subsection{The Newton-Raphson method}

The historical approach [12, 34, 37, 40, 42-47] involvesthe resolution 
of Comput Geosci

the 3.1).

syste

m (6)

with

the

202

However, many authors [18, 32, 38, 39, 48] have

Newt

on-

Raph

son

meth

od pro- 203 posed an alternative approach. Instead of using the com- 204 ponent concentrations $X_{j}$ as the primary variables, they 205

use the logarithm of the component activities (

$\ln X_{j}$ ). According to this convention, the objective

using

[X $]$

and

[Cp $]$

as

prim

ary

unkn

owns

This

Q3 func- 207

tions defined by Eq. 8 become conservation equations

208

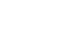

$Y_{j}=-T_{j}+{ }_{i=1}^{N_{C}} a_{i, j} \cdot \underline{K}_{V_{i}}^{\gamma_{i}} \cdot \exp { }_{k=1}^{N x} a_{i, k} \cdot \xi_{k}+{ }_{i=1}^{N_{C P}} a p_{i, j} \cdot\left[C p_{i}\right]$ for $j=1$ to $N_{x}$ 
209 In the case of the objective function describing precipitation,

210 it is more interesting to rewrite the mass action law (2) in

$211 \log$ form and then define the objective function

$$
Y_{N x+i}=\ln \left(\mathrm{SI}_{i}\right)=\ln \left(K p_{i}\right)+{ }_{k=1}^{N x} a p_{i, k} \cdot \xi_{k} \text { for } i=1 \text { to } N_{C P}
$$

Equations 8 and 9 are solved at the $n$th iteration with the Jacobian matrix $\left(Z^{n}\right)$ of the objective functions

$$
\begin{aligned}
& Z^{n}: j=1, N+N=\underset{+j i n}{\partial Y^{n}}
\end{aligned}
$$

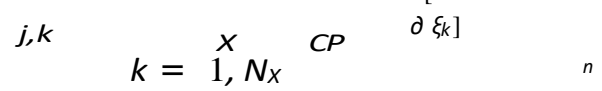

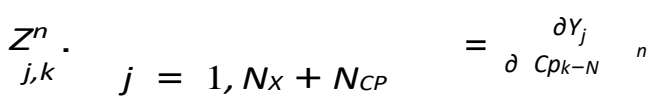

$$
\begin{aligned}
& k=N_{x}+1, N_{x}+N_{C P}
\end{aligned}
$$

$Z^{n}$ can be calculated in two ways.

(i) Using an analytical computation, we obtain the $\left(N_{x}+\right.$ $\left.N_{C P}\right) \times\left(N_{X}+N_{C P}\right)$ values of $Z^{n}$ by

$$
\begin{aligned}
& Z, \dot{j} \dot{j}=, N_{x}={ }^{N C} a_{i, j} \cdot a_{i, k} \cdot\left[C_{i}\right]^{n} \\
& j k \cdot k=1, N_{x} \quad i=1 \quad=a p_{k-N_{x, j}} \\
& Z^{n} \\
& j=1, N_{x} \\
& k=N_{X}+1, N_{X}+N_{C P} \\
& Z_{j, k}^{n} \cdot j=N_{x}+1, N_{x}+N_{C P}=a p_{k, j-N x} \\
& \begin{array}{c}
Z_{n, k} \cdot j=N_{x}^{k=1}+N_{x} \\
j=N_{x}+N_{C P}=0
\end{array}
\end{aligned}
$$

\subsection{Chemical test cases}

We choose chemical test cases with various numbers of 232 components. Some of these chemical systems allow the formation of mineral species. Although it is not realistic from a chemical point of view, we test them without minerals and with the maximal possible number of minerals to obtain the largest matrix size. Appendix 1 presents the stoichiometric coefficients, equilibrium constants, and concentrations for these tests.

(i) The gallic acid test case was presented by Bras- 240sard and Bodurtha [49]. It has been recognized as a 241 challenging test for speciation computation [17] (see 242

Appendix 1 (1)).

(ii) The Valocchi test is from Valocchi et al. [11]. It involves calcium and magnesium ion exchange (see Appendix 1 (2)).

(iii) The pyrite test case describes the dissolution of a 247 pyrite rock in pure water. It has been used to test spe- 248 ciation algorithms [17]. Because it involves redox 249 reactions, the stoichiometric coefficients cover a 250 wide range, and the equilibrium constants vary over 251 several orders of magnitude. This test is used under 252 Appendix 1.(3)) the assumption that no mineral phase is present (see (iv) The MoMaS easy test is the chemical system used

for the reactive transport benchmark of MoMaS at the mags leyel [50].It has feen specifically developed

(v) The Appendix 1 1 (4)) test is the first large chemical 259

$$
k=N_{x}+1, N_{x}+N_{C P}
$$

Even if the activity coefficients depend on the compo-nent concentrations, they are assumed to be constant during the NewtonRaphson procedure. These activity coefficientsare usually actualized by a fixed-point algorithm at each Newton-Raphson loop.

The progress step of the method $\left(\Delta \xi^{n}, \Delta C p^{n}\right)$ is achieved by assuming that the objective function $\gamma^{n+1}$ inEq. 12 is equal to zero at the $(n 1)$ th iteration. This pro-duces the key equation of this article, the linear system (12), which must be solved to obtain the progress step

$Z^{n} \cdot \Delta \xi^{n}, \Delta C p^{n}=\gamma^{n+1}-\gamma^{n}=-\gamma^{n}$

This system yields the values of the component activitiesand precipitate concentrations at the $(n+1)$ th iteration

$\xi^{n+1}=\xi^{n}+\Delta \xi^{n}$

$[C p]^{n+1}=[C p]^{n}+\Delta C p^{n}$

To simplify the notations, $\xi$ is used to denote the full vectorof unknowns, 
inclu ding mine ral $C p$ if prese nt.
Comput G was used by F. Morel and M. Morgan in 1972 to 262 present the capacities of the computational method 263 they had just developed (and which we still use 264 today). This test includes 52 components $\left(\mathrm{H}^{+}, 20265\right.$

metals, and 31 ligands), leading to 781 aqueous 266

species

(see

Appendix

1

(5)).

267

(vi) The MoMaS medium test is the chemical system for 268

the medium level of the MoMaS reactive transport 269

benchmark [50] (see Appendix 1 (6)).

270

(vii) The $\mathrm{Fe}-\mathrm{Cr}$ test is an additional redox test that 271

describes the redox reactions between iron and 272

chromium. These types of reactions occur when 273

iron reactive barriers are used to treat chromium- 274 contaminated sites [51, 52].

In this case, we con- 275 sider only the aqueous phase without minerals (see 276

Appendix

(7)).

\section{7}

(viii) The pyrite mineral test describes the dissolution of 278

a pyrite rock in pure water. We assume that three

$$
9
$$

possible mineral phases are present (see Appendix 1

(8)). 
(ix) The MoMaS hard test is the equilibrium part of the chemical system described in the hard level of the MoMaS reactive transport benchmark. It allows for the formation of two mineral species (see Appendix $1(9))$.

(x) The $\mathrm{Fe}$ - $\mathrm{Cr}$ mineral test describes the redox reaction between iron and chromium. We assume the formation of three different mineral phases (see Appendix 1 (10)).

\subsection{Test procedure}

Equation 11 shows that we can obtain multiple linear systems from one chemical problem by changing the activity values of the components. For each chemical system, we select three components and vary their values over a wide range. The concentrations of all minerals are arbitrarily set to $10^{-3} \mathrm{~mol} \mathrm{~L}^{-1}$. The activity of component $\mathrm{H}^{+}$is varied from $10^{-12}$ to $10^{-2} \mathrm{~mol} \mathrm{~L}^{-1}$ ( $\mathrm{pH}=12$ to $\mathrm{pH}=2$ ), while that of component $e^{-}$is varied from $10^{-19}$ to $10^{12}$, corresponding to $\mathrm{Eh}=0.7$ to $1.1 \mathrm{~V}$ computed using Eq. 14 at 25 ${ }^{\circ} \mathrm{C}$

$\mathrm{Eh}=\ln e^{-} \frac{R T}{F}$

where $T$ is the temperature (Kelvin), $R$ is the gas constant (8.314 $\left.\mathrm{J} \mathrm{K} \mathrm{mol}^{-1}\right)$, and $F$ is the Faraday constant $(96,487$ $\left.\mathrm{C} \mathrm{mol}^{-1}\right)$. This range of electrical potential corresponds to the stability of water at $\mathrm{pH}$ values between 2 and 12. For the $\mathrm{O}_{2}$ component, it is not possible to cover the same potential range as $e^{-}$because of the computation of the reference solution. The activity is varied from $10^{-70}$ to $10^{4}$, as com-

puted using Eq. 15 at $25{ }^{\circ} \mathrm{C}$ with $E^{0}=1.23 \mathrm{~V}$ and $\mathrm{pH}$ varying from 2 to 12 . The potential is then varied from -0.5

\section{to $1.1 \mathrm{~V}$}

$\mathrm{Eh}=E^{0}+1 R T \times \ln \underline{\left.\left\{\mathrm{O}_{2}\right\} \mathrm{H}^{+}\right\}_{4}}$

$$
4 F \quad\left\{\mathrm{H}_{2} \mathrm{O}\right\}
$$

The activities of the other components vary from $10^{-12}$ to $10^{-1}$ mol L ${ }^{-1}$. For each of the three selected components, we compute 30 values equally distributed on a log scale over

$$
\begin{aligned}
& 319 \\
& 320 \\
& 321 \\
& 322
\end{aligned}
$$

The condition number of $Z$ is defined [23] as the product of 323 the norm of the matrix per the norm of the inverse matrix 324 (17)

$\operatorname{cond}(Z)=\|Z\|_{1} \times{ }^{\cdot \cdot} Z^{-1 \cdot \cdot}$

$$
1
$$

To test the numerical methods, we first evaluate the compu- 326 tation time (CPU time) required to solve the linear system. 327 Because we work with a very small matrix, the computa- 328 tions are very fast and we run the same calculation several 329 times to obtain a total computing time of approximately 1330

s. The CPU time is given in this work in units of seconds per computation (by dividing the total computing time by the number of runs). According to this method, the global computing time for one test case is approximately 6 days.

Many numerical methods, including a failure indicator, which indicates the success or failure of the resolution, have 336 been developed. If needed, we include a failure indicator. 337 As failure, we include the crash of the method, underflow 338 or overflow, non-convergence within the maximum number 339 of iterations (for iterative methods), or excessive inaccu- 340 racy for some advanced methods (LAPACK routines) that 341 estimate the accuracy of the proposed solution. 342 Solving a linear system (13) using a numerical method 343 produces an approximate solution ( $\left.d \xi_{\text {method }}\right)$, and the ref- 344 erence method gives $\left(d \xi_{\text {ref }}\right)$ with accuracy on the same 345 order as the roundoff error. To evaluate the accuracy of the 346 approximate solution, two quantities can be calculated: $\quad 347$

1. The relative error on the norm, $\operatorname{Err}_{\mathrm{Norm}}$, is obtained by 348 computing the norm of the approximate and reference 349 solution (18)

$\operatorname{Err}_{\text {Norm }}=\frac{\left\|d \xi_{\text {method }}\right\|-\left\|d \xi_{\text {ref }}\right\|}{\left\|d \xi_{\text {ref }}\right\|}$

1. The error on the direction is given by angle method, the 351 angle (degrees) between the reference and the approx- 352 imate solution calculated using the scalar product of 353

these two vectors

354

$$
\begin{aligned}
& \text { angle } \quad=\quad \underline{360}^{A r c \cos } \quad \underline{d \xi_{\text {method }} \cdot d \xi_{\text {ref }}} \\
& \text { method } 2 \pi \quad\left\|d \xi_{\text {method }}\right\| \cdot\left\|d \xi_{\text {ref }}\right\|
\end{aligned}
$$

the chosen range, leading to 29,791 different linear systems for each chemical test case. For each of these 29,791 tests, we make only one linear solver (or one Newton step) (exceptin the last section, Section 4, where the iterative Newton method is performed to solve the non-linear system given 
by Cgmput Geesci quantities, namely the failure indicator

Eqs. relative 355 error on the norm, angle $e_{\text {method, }}$ and CPU

8 and time, are calculated 356for the 29,791 linear systems

9). built from each chemical test 357 case for all the

$T$ tested methods. This enormous amount of $35 \mathrm{k}$ d Ata

he is aggregated in two

matri ways:

$\mathrm{X}$

norm 359

used (i) For each chemical system and each method, we in com-

this $\quad 360$

work pute the mean of each

is the quantity.

1

norm

,

defin

ed as

[23]

361

(ii) For each chemical system and each method, the inter-

362

val of the condition number is discretized into

100

$\|Z\|_{1} \stackrel{363}{\nexists \max } \cdot Z_{i, j}$.

reg변 $\underline{a}_{n}$ subintervals. For each subinterval, we compute 364

the mean of each quantity.

$i=1$ 


\subsection{Reference solution}

Because of the very high condition numbers, it is not possible to directly obtain an exact solution. We equilibrate the rows and columns of the Jacobian matrices to reduce their condition number using the iterative algorithm proposed by Knight et al. [53] because it preserves the symmetry of the Jacobian matrix.

$\widetilde{{ }^{k}}{ }^{k}$ be the equilibrated Jacobian matrix at iteration $k$,
withe $\mathbf{Z}^{\boldsymbol{Z}}=Z$.
These authors defined $r_{i}^{k}$ as the vector formed by the ith
$k$

row of $\widetilde{\mathbf{Z}}$ and $c_{i}^{k}$ as the vector formed by the $i$ th column. The preconditioning matrices $\mathbf{R}^{k}$ and $\mathbf{C}^{k}$ are then defined by
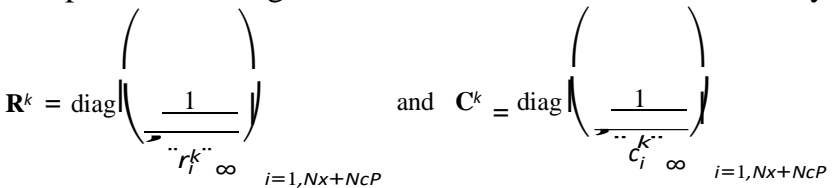

The equilibrated matrix is defined at iteration $k+1$ by

$\widetilde{\mathbf{Z}}^{k+1}=\mathbf{R}^{k} \cdot \widetilde{\mathbf{Z}}^{k} \cdot \mathbf{C}^{k}$

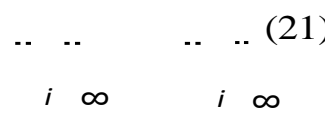

equal to 1 or after 50 iterations. Let $\mathbf{R}$ and $\mathbf{C}$

This procedure is repeated until all " $r^{k "}$ and " $c^{k}$ are

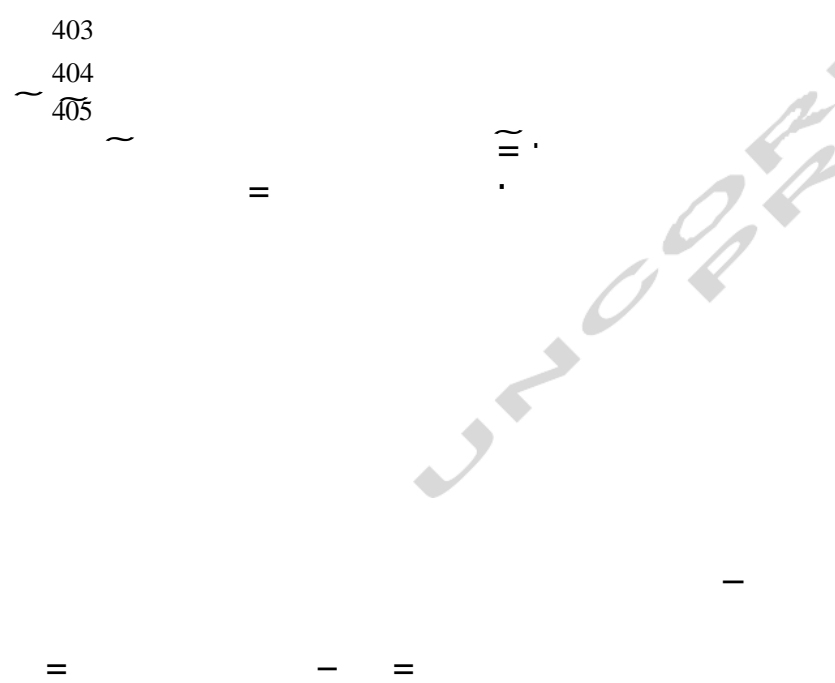

methods, such as Gaussian elimination [34] or LU decomposition [17, 40, 42]. In its actual form, the speciation code SPECY [48] uses unsymmetric multifrontal (UMF) [55] as the linear solver. To the best of our knowledge, no speciation code uses iterative methods to solve linear systems. This point is in accordance with the existing literature, which reports the use of iterative methods for solving large, sparse linear systems [20-22, 24, 26, 28, 29, 56, 57]. Nevertheless, 413 actual developments in speciation codes involve the use of 414 large chemical databases [39, 58, 59], leading to an increase 415in the size of the chemical systems. The use of iterative 416 methods is also studied in this work.

We select some direct and iterative solvers according 418

to the properties of the linear systems and the speciation 419 computation methods currently in use (Table 1). 420 Q6

For the direct method, we select LU decomposition [60] 421 because it was originally used for speciation computa- 422 tions by Westall [40] and Westall et al. [42]. The UMF 423 method [55] has been implemented in the speciation code 424 SPECY [48] in place of the LU approach [17]. After show- 425

ing that the Jacobian matrix is symmetric, we test the 426

DSYTRS subroutine from LAPACK [61], which is based 427 on a UDU decomposition. Because the Jacobian matrix is 428

DPOTRS subroutine [61] based on the Cholesky method. 430

often positive definite, as shown in Table 3, we test the 429

ing preconditioning matrices and $\mathbf{Z}$ the equilibrated matrix. be the resultInstead of solving the linear system (12), we solve

$\mathbf{Z} \cdot \mathbf{x}=-\mathbf{Y}$

where $\mathbf{x} \mathbf{C}^{-1}(\Delta \xi, \Delta C p)$ and $\mathbf{Y} \quad \mathbf{R} \quad Y$. These proce- dures are coded using quadruple-precision reals. The linearsystem (22) is solved by LU decomposition coded with quadruple-precision real.

Even if the condition numbers of the Jacobian matrices (Z) are very high $\left(10^{213.9}\right.$ for the Fe-Cr mineral test case), the condition numbers of the equilibrated matrices $(\mathbf{Z})$ are much lower: the maximum condition number obtained afterequilibration is $10^{13.4}$. According to Golub and van Loan [54], if the unit roundoff is approximately $10^{-d}$ and the condition number is approximately $10^{q}$, then the Gaussian elimination gives a solution with approximately $d q$ cor- rect digits. Because we use quadruple precision, we obtain $d 32$, leading to 321418 correct digits. One can thenassume that the reference solution is exact if we compare it to the solutions produced by the tested methods (computed using doubleprecision real).

\subsection{Selected numerical methods for solving linearsystems}

Studies on linear algebra [19, 23] present methods for solving linear systems as direct or iterative methods. Histor-ically, speciation codes solved linear systems using direct 
Comput Gegsci

$[23$,

434

62], Gauss-Seidel [23, 62], and successive over-

relaxation

435

(SOR) [23, 62] methods. Barrett et al. [21] proposed an

436

algorithm to select an iterative solver depending on the

437

matrix properties. GMRES was presented as the least

selec-

438

tive algorithm. We use a GMRES method developed by

439

HSL [63]. If the matrix is symmetric, Barrett et al. [21]

rec-

440

ommend the use of conjugate gradient squared (CGS)

or

441

biconjugate gradient stabilized (BiCGStab) methods.

CGS

442

and BiCGStab subroutines have been developed by

HSL. 443 We test two additional methods devoted to symmetric matri- 444ces: SYMMBK [63] and an incomplete Cholesky (Inc. 445 CHOLESKY) factorization

[63].

446

We use the same parameters for all iterative methods:

a 447

maximum of 500 iterations and a stopping criterion of $10^{-8}$. 448

To determine the influence of the stopping criterion, we test

449

the GMRES method using 50,000 maximum iterations

and

450

$10^{-12}$ as the stopping criterion, denoted by GMRES $10^{-12}$ 451

in this study. A critical point of the GMRES algorithm is the

452

祭脑eriog the Hessenberg matrix. In this work, we set it to the

$\max$ of $8(\mathrm{Nx}+\mathrm{NcP})$.

The results obtained using the Jacobi and SOR methods are not detailed here. As previously reported [19], the Jacobi method is inefficient, leading to a very high failure ratio 
Table 1 List of the selected solvers
Table 2 Structure of the Jacobian matrix

\begin{tabular}{|c|c|c|c|}
\hline Name & Source & Method & Matrix properties \\
\hline \multicolumn{4}{|l|}{ Direct } \\
\hline LU & {$[58]$} & LU decomposition & - \\
\hline DGETRS & [59] & LU decomposition & - \\
\hline UMF & {$[53]$} & Direct multifrontal & - \\
\hline DSYTRS & [59] & UDU-factored symmetric matrix & Symmetric \\
\hline DPOTRS & [59] & Cholesky $\mathrm{A}=U^{\mathrm{T}} \times U$ & Definite positive \\
\hline DGELS & [59] & QR decomposition & \\
\hline LU QUAD & {$[58]$} & LU decomposition quadruple precision & - \\
\hline \multicolumn{4}{|l|}{ Iterative } \\
\hline SYMMBK & {$[61]$} & Iterative SYMMBK HLS_MI02 & Symmetric \\
\hline Inc. CHOLESKY & {$[61]$} & Incomplete Cholesky HSL_MI28 & Symmetric \\
\hline CGS & {$[61]$} & Conjugate gradient squared HLS_MI23 & - \\
\hline BiCGStab & {$[61]$} & Biconjugate gradient squared stabilized HLS_MI26 - & - \\
\hline GMRES & [61] & Flexible GMRES HLS_MI15 & - \\
\hline Gauss-Seidel & {$[58]$} & Gauss-Seidel method & - \\
\hline \multicolumn{4}{|l|}{ Preconditioned } \\
\hline LU Equil & [51-58] & LU and matrix equilibration & - \\
\hline DGESVX & [59] & LU and optional preconditioning & - \\
\hline GMRES Equil & [51-61] & GMRES and matrix equilibration & - \\
\hline GMRES 1.d-15 & {$[61]$} & GMRES convergence criteria 1.d-15 & - \\
\hline
\end{tabular}

method [23, 26, 56, 62], the over-relaxation parameter is the key factor. Unfortunately, we did not find any efficient relationships to define it. For the same chemical system, the best value varies from 0.097 to 1.91 without apparent order.

We do not extensively test the possibility of using a preconditioner. As stated by Barrett et al. [21]: "Since using a preconditioner in an iterative method incurs some extra cost, both initially for the setup, and per iteration for applying it, there is a trade-off between the cost of constructing and applying the preconditioner, and the gain in convergence speed". In our case, the matrices are very small, leading us to suppose that this trade-off would not be advantageous. Nevertheless, an easy way to test preconditioners is proposed by the LAPACK routine DGESVX, which performs LU decomposition and matrix equilibration depending on the estimated condition number. We implement matrix equilibration according to Knight et al. [53] to obtain a reference solution. We test this preconditioning technique associated with LU decomposition and the GMRES method, denoted by LU Equil and GMRES Equil in this study. The maximum iterations allowed for the equilibration procedure is fixed to 5 , according to the recommendations of Knight et al.
470

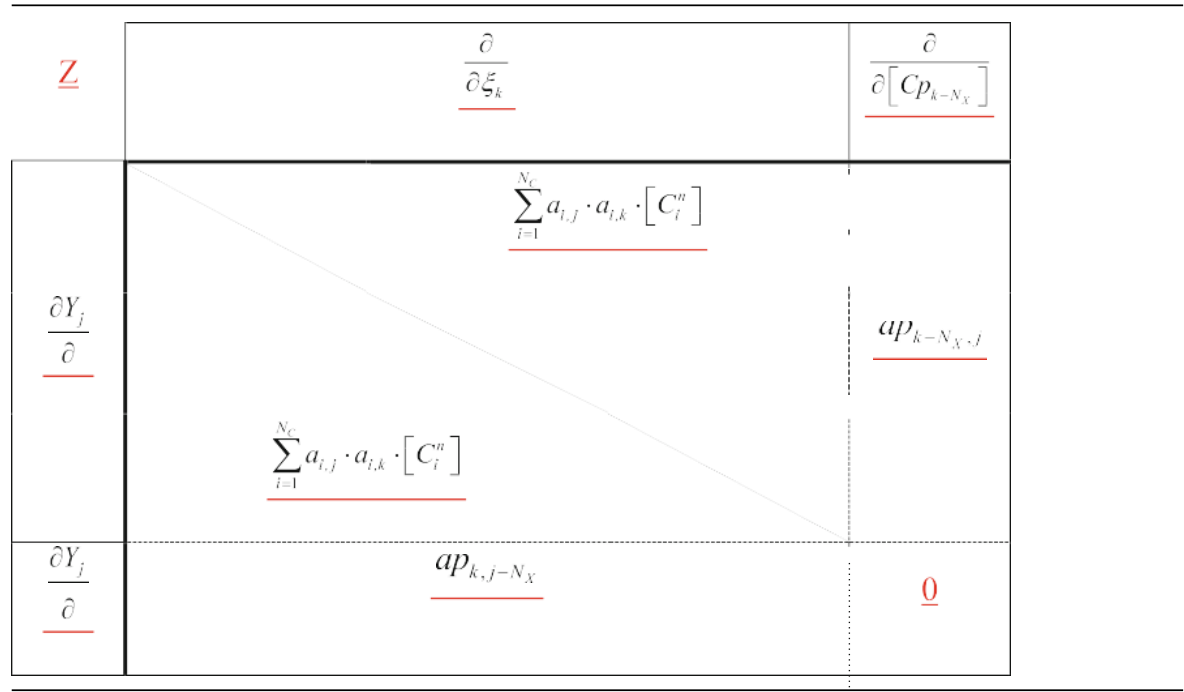


Table 3 Properties of the 10 chemical test cases ranked by increasing the maximal condition number

\begin{tabular}{|c|c|c|c|c|c|c|c|c|}
\hline $\mathrm{Nx}$ & $\mathrm{Nc}$ & $\mathrm{NcP}$ & $Z$ size & $\operatorname{cond}(Z) \min$ & $\operatorname{cond}(Z) \max$ & $\begin{array}{l}\operatorname{cond}(Z) \\
\max \text { after } \\
20 \text { equili- } \\
\text { bration }\end{array}$ & $\begin{array}{l}\% Z \text { diag- } \\
\text { onal domi- } \\
\text { nant }\end{array}$ & $\begin{array}{l}\% Z \text { positive } \\
\text { definite }\end{array}$ \\
\hline
\end{tabular}

\begin{tabular}{llllllllll}
\hline Gallic acid & 3 & 17 & 0 & 3 & $10^{0.61}$ & $10^{12.6}$ & $10^{0.95}$ & 18.4 & 100 \\
Valocchi & 5 & 7 & 0 & 5 & $10^{0.49}$ & $10^{15.3}$ & $10^{0.65}$ & 67.7 & 100 \\
Pyrite & 4 & 40 & 0 & 4 & $10^{4.06}$ & $10^{24.9}$ & $10^{0.95}$ & 0.00 & 100 \\
MoMaS easy & 5 & 12 & 0 & 5 & $10^{3.44}$ & $10^{37.7}$ & $10^{1.05}$ & 0.00 & 71.1 \\
Morel-Morgan & 52 & 781 & 0 & 52 & $10^{43.4}$ & $10^{60.7}$ & $10^{1.13}$ & 0.00 & 35.9 \\
MoMaS medium & 5 & 14 & 0 & 5 & $10^{5.88}$ & $10^{103.9}$ & $10^{0.95}$ & 0.00 & 78.8 \\
Fe-Cr & 7 & 39 & 0 & 7 & $10^{9.46}$ & $10^{113.6}$ & $10^{1.05}$ & 0.00 & 68.9 \\
Pyrite mineral & 4 & 43 & 3 & 7 & $10^{1.71}$ & $10^{33.1}$ & $10^{3.19}$ & 0.00 & 0.00 \\
MoMaS hard & 6 & 15 & 2 & 8 & $10^{5.45}$ & $10^{123.9}$ & $10^{3.02}$ & 0.00 & 0.00 \\
Fe-Cr mineral & 7 & 43 & 3 & 10 & $10^{8.67}$ & $10^{213.9}$ & $10^{13.4}$ & 0.00 & 0.00 \\
\hline
\end{tabular}

$d \xi_{\text {EU QUAD }}$
Finally, we test an LU decomposition method compiled as quadruple precision, denoted by LU QUAD. The source of this method is the LU double-precision real of numerical recipes [60], and we adapt it to quadruple precision. Because the usual computations are performed using double precision, the quadruple precision ( $\left.d \xi_{\mathrm{QUAD}}\right)$ should be translated in double-precision real. To avoid overflow, we rescale $d \xi_{\mathrm{QUAD}}$ to ensure its validity. If huge (1.d0) is the highest double-precision real represented by the machine, we rescale $d \xi_{\mathrm{QUAD}}$ to obtain the double-precision solution $d \xi_{\text {LU QUAD: }}$

$\cdot d \xi_{\text {QUAD }}$

$$
d \xi_{\mathrm{QUAD}} i()
$$

In this way, we conserve the direction of the Newton step,

\section{Results and discussion}

\subsection{Properties of the Jacobian matrices}

As defined by Eq. 11, the Jacobian matrix has several properties:

(i) The matrix is block-structured, as presented in Table 2. A four-block structure is present if precipitation occurs.

(ii) The matrix is symmetric, as shown in Table 2.

(iii) In the case of no precipitation, all the diagonal terms of the matrix are strictly positive because they are the sum of $a_{i, j}^{2}\left[C_{i}\right]$. It is then possible for the matrix to be diagonal dominant. We examine this possibility for the selected test case. Table 3 shows the ratio of diagonal dominant Jacobian matrices for all the chemical tests performed according to the previously defined test procedure. Some matrices in the gallic acid and Valocchi cases are diagonal dominant, but none of the matrices from the other cases are diagonal dominant. By plotting the ratio of diagonal dominant matrices depending on the condition number (see Appendix 2 (B-1)), it appears that only matrices with very low condition numbers can be diagonal dominant.

(iv) Because the Jacobian matrix is real, symmetric, and sometimes diagonal dominant, the question of whether it is positive definite may be posed. In the case of no precipitation, Eq. 11 can be written in matrix form, leading to Eq. 24

$Z=A^{T} \cdot \operatorname{diag}(C) \cdot A$

Because the concentrations are positive, the Jacobian matrix 521is analytically positive definite. Nevertheless, this may not 522 be true numerically. We are not able to propose a gen- 523eral framework, but we can compute the eigenvalues of the 524 Jacobian matrix and test whether they are positive for all 525 test cases. Table 3 shows that for the gallic acid, Valoc- 526chi, pyrite, and Morel-Morgan test cases, all the Jacobian 527 matrices are positive definite. For the MoMaS easy, MoMaS 528medium, and $\mathrm{Fe}-\mathrm{Cr}$ test cases, a large proportion (66.4 to 529

$74.1 \%$ ) of the Jacobian matrices are positive definite. For 530 cases including minerals (pyrite mineral, MoMaS hard, and $531 \mathrm{Fe}-$ $\mathrm{Cr}$ ), essentially none of the matrices are positive defi- 532 nite (only $0.1 \%$ for the MoMaS hard test). Plotting the ratio 533of positive definite matrices as a function of the condition 534 number (see Appendix 2 (B-2)) shows that the chemical 535 conditions are more important than the condition number 536 when determining whether the Jacobian matrix is diagonal 537 dominant.

538

(v) According to the test procedure presented previously, 539 we plot, on the same graph, the logarithm of the norm $\quad 540$ 
of I Ind the logarithm of the condition number of the matrix Z (Fig. 1). There is a strong linear relation-ship between these parameters. Moreover, the linear relationship does not depend on the chemical test, only on the existence of minerals. According to our results, the conditioning of the $\mathrm{Z}$ matrix can be evaluated using the following empirical formulas:

cond $(Z)_{\text {no mineral }}=10^{5.30 \pm 0.03} \times\|Y\|^{0.9374 \pm 0.0008}$ cond $(Z)_{\text {mineral }}=10^{-3.23 \pm 0.08} \times\|Y\|^{1.706 \pm 0.002}$

The value and uncertainties are obtained through the least squares method over all $\operatorname{cond}(Z)$ and ||$Y|$.$| In this way, we$ propose an estimation of $\operatorname{cond}(Z)$ with no computation time cost because the objective function is evaluated during the Newton-Raphson procedure. As shown in Fig. 1, cond( $Z$ ) and $\|Y\|$ are strongly correlated for large condition num- bers, and the results are noisier if $\operatorname{cond}(Z)$ and $\|Y\|$ are small. The evolution of this relation for low |Y ||can be seen $555 \mathrm{in}$ Appendix 7 (G-11). Therefore, Eq. 25 should not be used 556for $Y$ |lles ||s than $10^{10}$.

Several of these properties are obtained using the logarithm of the component activities as the primary unknown in Eq. 8. The historical approach [34] uses the component concentrations as the primary variable and leads to a less interesting Jacobian matrix. Even if the structure presented 562 in Table 2 exists, the matrix is not symmetric. Moreover, 563 the matrix is worse conditioned (condition number from $56410^{11.2}$ to $10^{49.4}$ rather than $10^{4.06}$ to $10^{24.9}$ for the pyrite 565

case). Finally, no specific relation exists between cond( $Z$ ) and $\|Y\|$ for the historical formulation.

As an example, we show one linear system from the Fe$\mathrm{Cr}$ mineral test, corresponding to a condition number of $10^{187}$. One can observe the structure of the matrix and the specificity of the linear system (26).
557

558

559

560

561 (2)

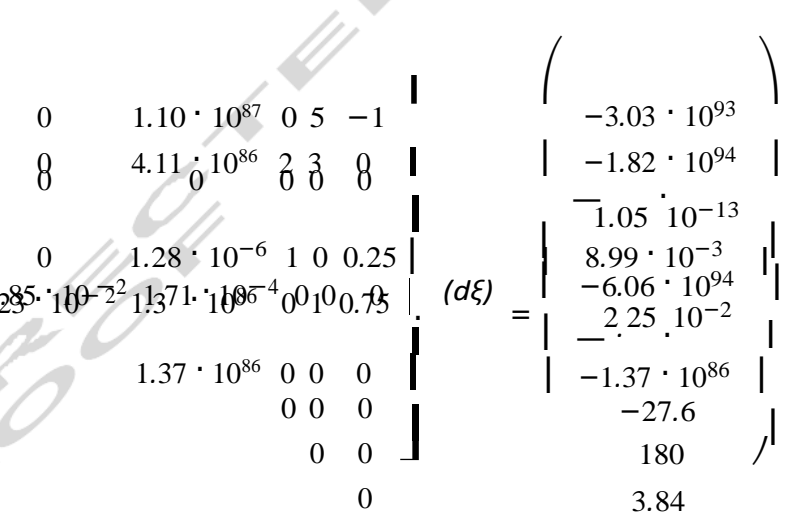

\subsection{Robustness of the methods}

Figure 2 presents the failure ratio for each method and each test case. The presence of minerals prevents the DPOTRS, Inc. CHOLESKY, and Gauss-Seidel methods from solving the system. If there are minerals present in the chemical system, a zero-value block appears in the Jacobian matrix, as shown in Table 2 and Eq. 26. This block makes the Inc. CHOLESKY factorization unappropriated. Because the Gauss-Seidel method requires division by each diagonal term, this zero-value block makes the method unadapted. The failure of the DPOTRS routine is explained by the properties of the Jacobian matrix. As shown in Table 3, there is no positive definite matrix in the presence of minerals. In the case of the DPOTRS, Inc. CHOLESKY, and Gauss-Seidel methods, the term failure is ambiguous. These methods are expected to fail and should not be used on systems with minerals. If there are no minerals, some matrices are not positive definite in the MoMaS easy, MoMaS medium, Morel-Morgan, and $\mathrm{Fe}-\mathrm{Cr}$ tests. This explains the
Some other methods (DGETRS, DSYTRS, DGELS, and DGESVX) present a substantial failure ratio, mainly for high condition number tests (MoMaS easy and $\mathrm{Fe}-\mathrm{Cr}$ mineral). UMF, SYMMBK, and CGS are robust for the $\mathrm{Fe}-\mathrm{Cr}$ mineral test but present significant failure ratios for lowerconditioned tests, such as MoMaS easy or pyrite mineral. Some methods adapted to symmetric matrices (DSYTRS and SYMMBK) are included in this class of weak methods.

The BiCGStab method has a very low failure ratio and fails only in the two difficult tests (MoMaS easy and $\mathrm{Fe}-\mathrm{Cr}$ mineral). GMRES is the only successful iterative method.

Figure 2 shows that some methods are successful for all the test cases. The most successful direct method is LU, while the most successful iterative methods are GMRES and GMRES $10^{-12}$. The quadruple-precision method LU QUAD is also successful, which is expected because the double-precision LU method is also successful. The use of an equilibration method as a preconditioner makes LU Equil and GMRES Equil successful.

As stated previously, we focus on the capacity of a 
Fig. 1 Relationship between the condition number of $Z$ and the norm of the objective function plotted on a log-log scale

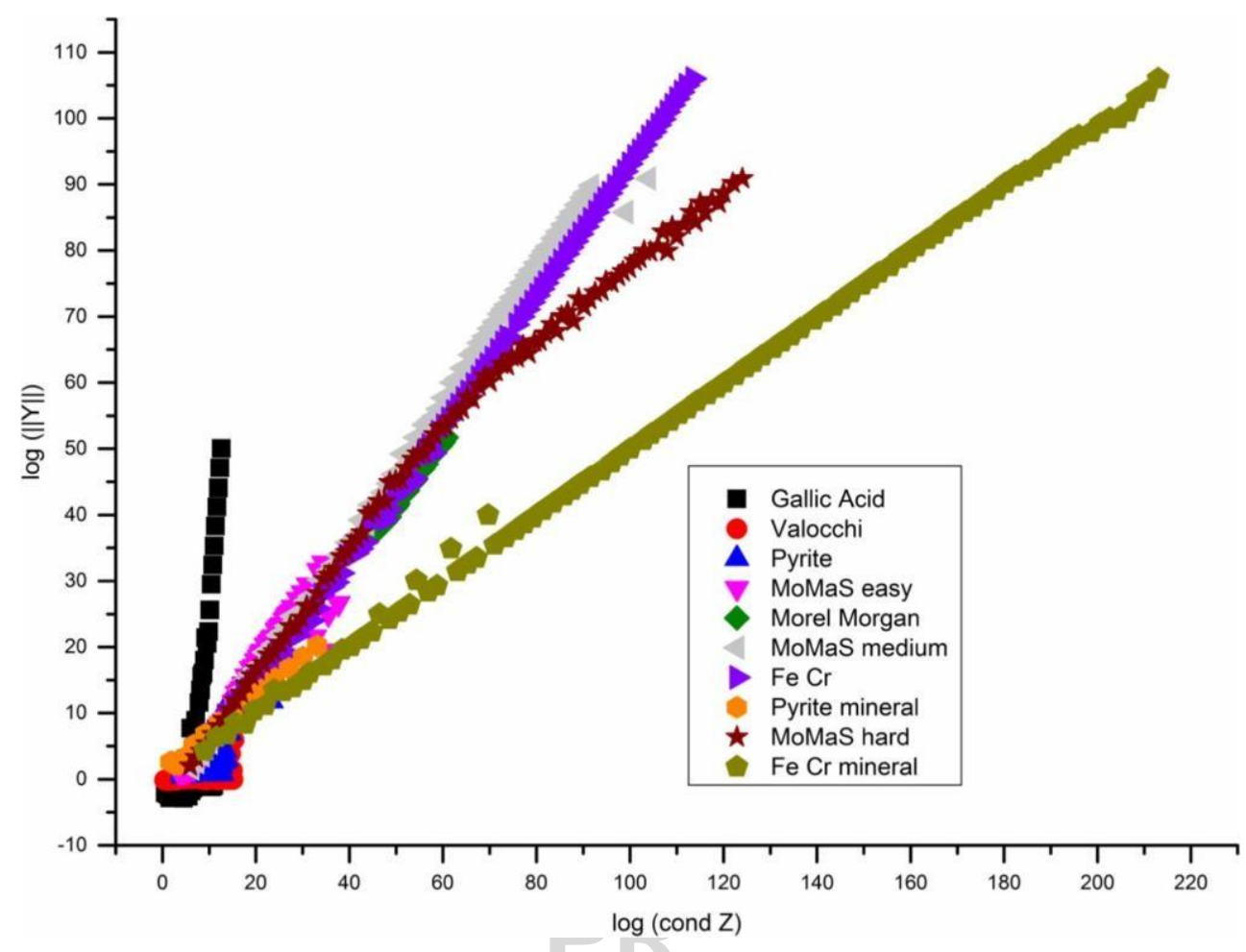

For some advanced methods (e.g. LAPACK methods), a posteriori estimation of the residual and estimation of the condition numbers are performed. If the solution is not sufficiently accurate, no solution is given, leading to a higher failure ratio than for the more rustic methods (LU or GaussSeidel). Because the key point of this work - the resolution of a linear system - is included in the iterative Newton procedure, it is preferable to obtain an inaccurate solution (so
617 618 619 620

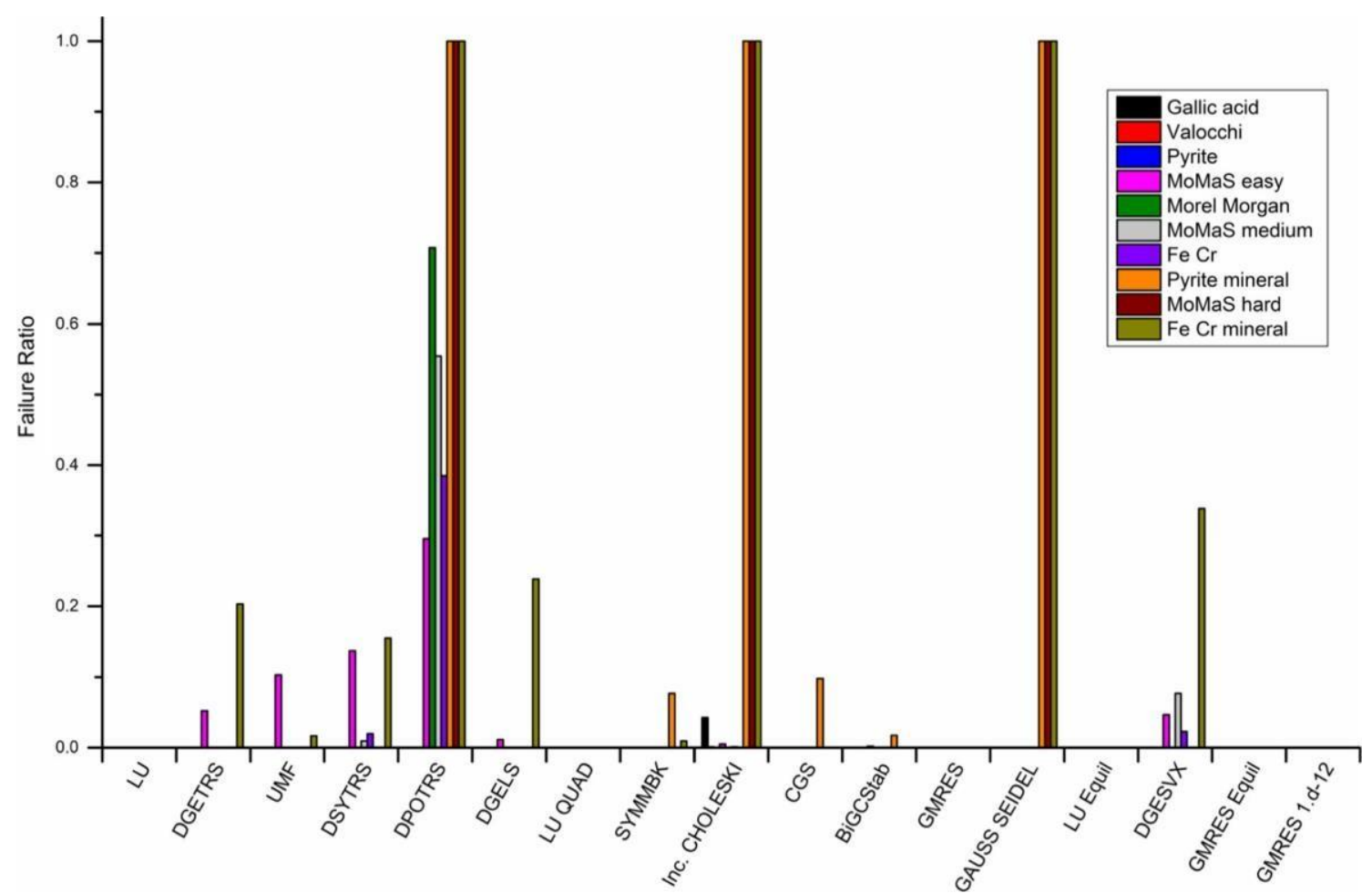

Fig. 2 Mean of the failure ratio for each method and each test case 
621 the iterative procedure can be continued) than no solution

(the iterative procedure will be aborted).

Appendix 3 presents the evolution of the failure ratio for each test case and each method depending on the condition number.

For the direct methods (Appendix 3 (C-1 to $\mathrm{C}-5)$ ), for small condition numbers corresponding to the test cases gallic acid, Valocchi, and pyrite, no failure occurs. As the condition number increases, the failure ratio also increases for some methods. MoMaS easy (Appendix 3 (C-4)), MoMaS medium (Appendix 3 (C-6)), and Fe-Cr (Appendix 3 (C7)) show that for condition numbers greater than $10^{20}$, the failure ratio increases greatly for some of the methods. These methods are DOPTRS and DSYTRS for MoMaS medium and Fe-Cr. DGETRS, UMF, DSYTRS, DOPTRS, and DGELS present some failure for condition numbers greater than $10^{15}$ for the MoMaS easy case. In the presence of minerals (Appendix 3 (C-8 and C-9)), for low condition numbers (the pyrite mineral case), the methods are either successful (UMF, LU, DSYTRS, DGETRS) or completely unsuccessful (DPOTRS). For very high condition numbers (Fe-Cr mineral case), the success of the method does not depend on the condition number. We suppose that the condition numbers (see Table 3 ) are too high to exhibit any ordering.
For other iterative methods, the success does not depend on the condition number but on the nature of the matrix and the presence (Appendix 3 (C-18 to $\mathrm{C}-20)$ ) or absence (Appendix 3 (C-11 to $\mathrm{C}-17)$ ) of minerals.

\subsection{Accuracy of the methods}

The accuracy of the methods is evaluated in two ways: (i) the relative error on the norm (18) and (ii) the angle between the reference and the calculated solution (19).

(i) By plotting the mean of the logs of the relative error 654on the norm of each test case (Fig. 3), some general 655 tendencies are identified. The relative residual tends to 656 increase with the condition number of the system. For 657 direct methods and small condition numbers, the relative residual is small $\left(10^{-10}\right.$ to $\left.10^{-3}\right)$ for the gallic acid, Valocchi, and pyrite test cases. For the iterative methods, the relative residual corresponding to an accurate resolution for tests with small condition numbers is approximately $10^{-4}$. This value corresponds to the value of the convergence criteria of the iterative methods. Iterative methods are more sensitive to the condition number than direct methods. Only the Valocchi test case is accurately solved by almost all the 658

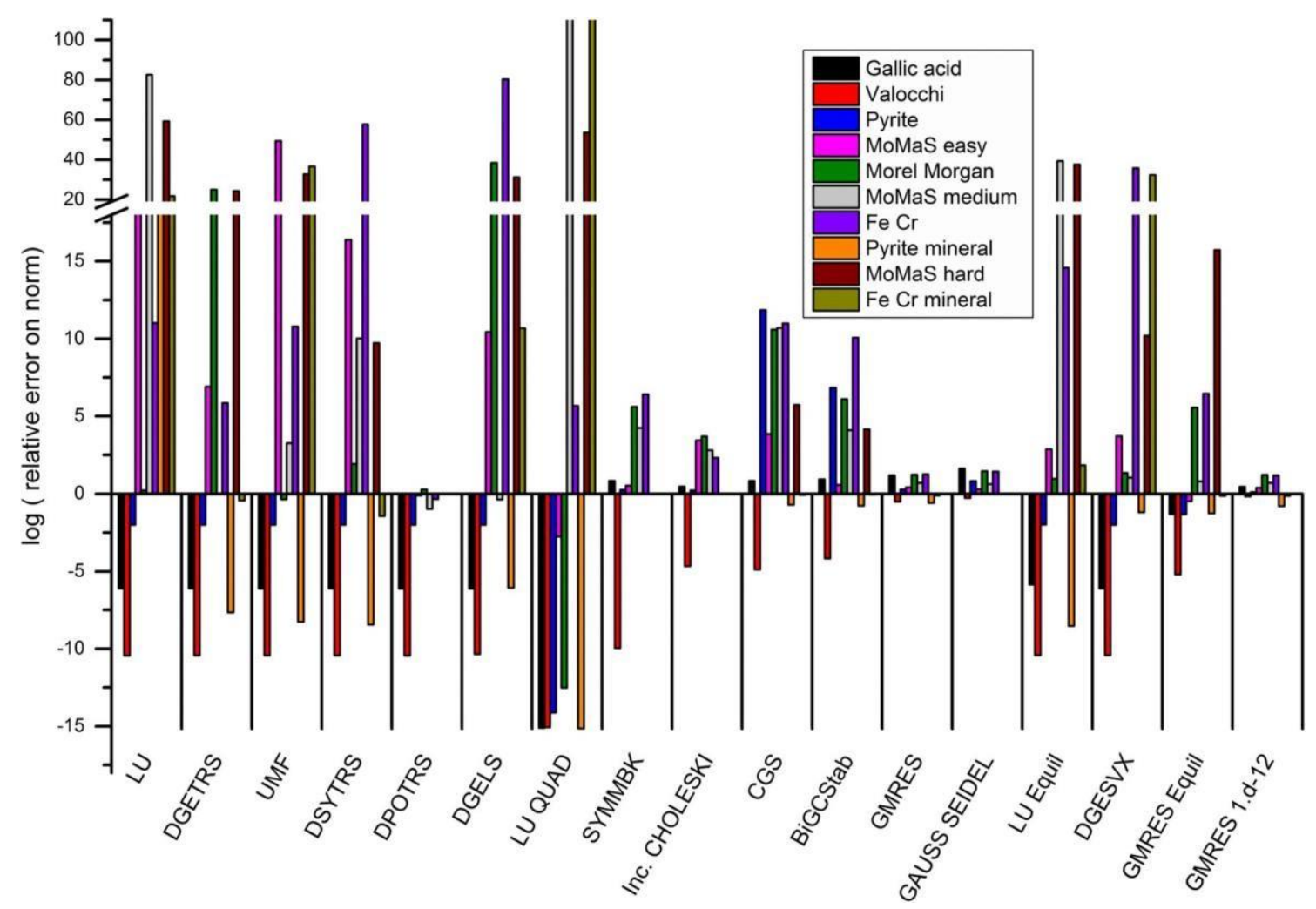

Fig. 3 Mean of the logs of the relative error on norm for each method and each test case 
iterative methods, whereas the first three tests are accurately solved by all the direct methods. Even in the case of successful resolution (CGS and BiCGStab methods), the relative errors on the norm are high for intermediate cases (pyrite, MoMaS easy, and Morel-Morgan). Nevertheless, the results are better for the iterative methods than for the direct methods for the difficult tests (MoMaS easy, MoMaS medium, MoMaS hard, FeCr mineral). The GMRES and Gauss-Seidel methods have mostly constant mean relative error on the norm, with the same accuracy for all test cases. GMRES and Gauss-Seidel are less efficient than the other methods for the easy tests, but more ill-conditioned tests are better solved by these two methods.

The condition numbers are so high that even LU QUAD cannot provide accurate resolution. For the MoMaS medium and $\mathrm{Fe}-\mathrm{Cr}$ mineral tests, many of the solutions calculated by the LU QUAD method are rescaled using Eq. 23, leading to excessively high relative error on the norm.

Comparison of the relative error on the norm given by the non-preconditioned (LU, DGETRS, and GMRES) and preconditioned (LU Equil, DGESVX, and GMRES Equil) methods shows that the preconditioned methods lead to lower relative error than the non-preconditioned methods for the direct methods, but the result is more case-dependent for GMRES. The use of preconditioning usually leads to lower relative error on the norm, except for the MorelMorgan, Fe-Cr, and MoMaS hard cases.

Increasing the maximum number of iterations and reducing the convergence criteria of GMRES leads to less relative error on the norm, but this reduction is not significant.

Nevertheless, the global means of the logs of relative errors on the norm hide the influence of the increasing condition number. Appendix 4 presents the evolution of the relative error on the norm for each test case and each method depending on the condition number. The theoretical behaviour is verified for the direct methods and for all the test cases (except for the Valocchi one, Appendix 4 (D2)). The relative error on the norm increases regularly with 706 the condition number. It is close to $10^{-16}$ when the con- 707 dition number is close to 1 and increases to 1 when the 708 condition number is close to $10^{16}$, in accordance with the 709 computation theory presented by Golub and van Loan [54]. 710

For condition numbers greater than $10^{16}$, the evolution of the relative error on the norm with the condition number is much noisier. The use of the quadruple-precision LU QUAD method leads to an accurate resolution of a large portion of the tested systems. As expected by computation theory, all the systems with condition numbers less than $10^{32}$ are .

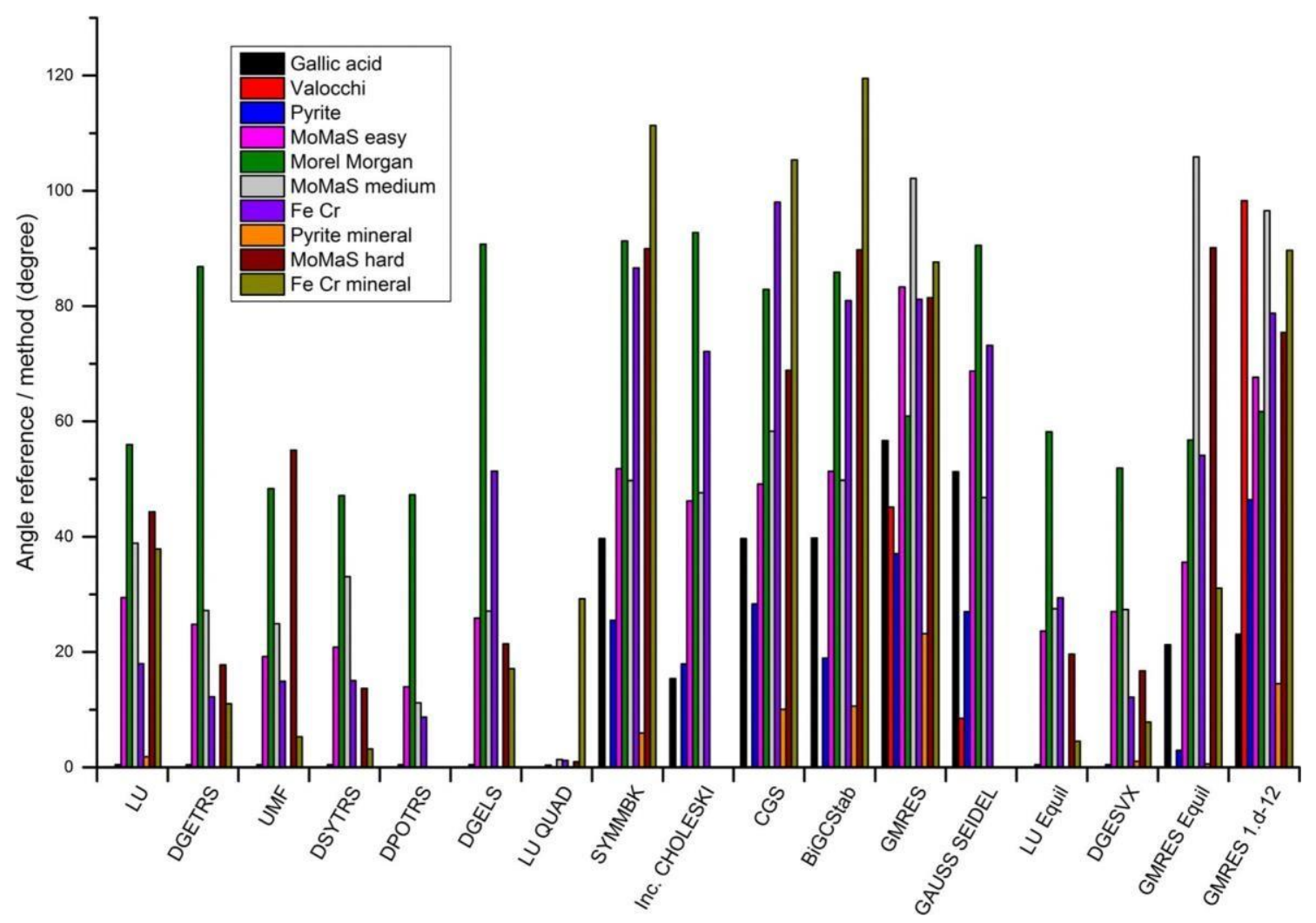

Fig. 4 Mean of the angles between reference and computed solution for each method and each test case 
Comput Geosci

Fig. 5 CPU time (s) for each method and each test case

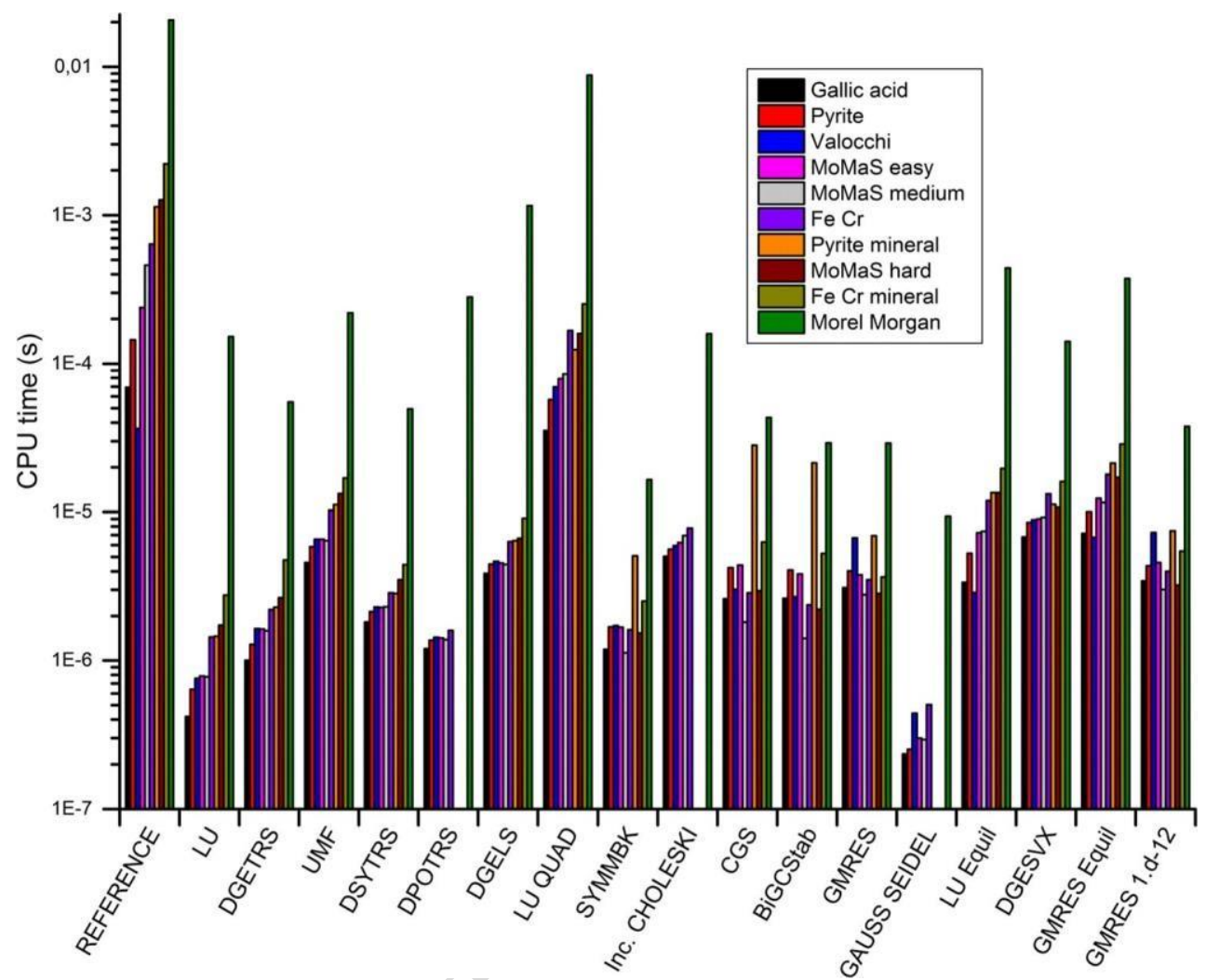

solved with a relative error on the norm of approximately $10^{-15}$. In some cases (MoMaS medium (Appendix 4 (D6)), Fe-Cr (Appendix 4 (D-7)), MoMaS hard (Appendix 4 (D-9))), LU QUAD produces an increasing relative error with increasing condition number (if higher than $10^{32}$ ) but not systematically. LU QUAD produces a very low relative error on the norm even if the condition number is very high (Appendix 4 (D-9)). This behaviour can be explained by the fact that the LU QUAD method and/or the reference method is unable to exactly solve such ill-conditioned systems. LU QUAD produces a very high relative error on the norm, one point with $10^{290}$ error for the MoMaS medium (Appendix 4 (D-6)), and all the values at condition numbers greater than $10^{90}$ for the Fe-Cr mineral (Appendix 4 (D-10)) test case. These points correspond to the rescaling of the computed quadruple-precision solution to maintain it on the double-precision scale (using Eq. (23)).

Iterative methods present similar behaviour to direct methods, giving very low relative error on the norm (between $10^{-15}$ and $10^{-8}$ ) when the condition number is

less than a critical value. This critical value depends on the

743 pyrite (Appendix 4 (D-13)), the MoMaS medium (Appendix 4 (D-16)), and MoMaS hard (Appendix 4 (D-19)) tests. Using low convergence criteria (GMRES 1.d-12) leads to lower relative error on the norm for low condition numbers (Appendix 4 (D-21 to D-23, D-26 to D-29)), but no significant improvements are obtained if the condition number increases, as shown in Appendix 4 (D-24 to D-30).

Using preconditioning methods reduces the relative error on the norm for intermediate condition numbers. No gain is obtained for low condition numbers (Appendix 4 (D-21 and D-22)), but the errors given by LU Equil, DGESVX, and GMRES Equil are less than the LU and GMRES errors for higher condition numbers (Appendix 4 (D-24 to D-26)). For very high condition number tests (Appendix 4 (D-27, D-29, and D-30)), the errors given by the preconditioned methods are equivalent to the errors given by the non-preconditioned methods.

744 745 746 747 748 749

(ii) By plotting the angle between the reference solu- 761 tion and the calculated solution, we can compare the 762 methods according to the computed direction (Fig. 4). 763

Because the resolution of the linear system (13) repre- $\quad 764$ Q8

method and the test case. It can be set to $10^{8}$ for SYMMBKCGS, BiCGStab, and GMRES for the gallic acid (Appendix4 (D-11)) and MoMaS easy (Appendix 4 (D-14)) cases.It can be set to $10^{12}$ or $10^{15}$ for Inc. CHOLESKY forthe gallic acid and MoMaS easy cases and for SYMMBK, Inc. CHOLESKY, CGS, BiCGStab, and 
RES this 765 information is much more important than for the norm 766of the step. A wrong norm can be the corrected using 767 line search methods [64], whereas modifying a wrong 768 direction leads to additional iterations. Small condition 769 number tests (gallic acid, Valocchi, pyrite, and pyrite 770 
Equil, LU Equil, and LU. Ranking these methods according 814 to the angle between the reference and computed solution is

mineral) are solved using direct methods with the right direction. If the condition number increases, the directions given by the direct methods become inaccurate, but the condition number is not the only govern- ing parameter. Morel-Morgan leads to worse directionthan MoMaS medium and $\mathrm{Fe}-\mathrm{Cr}$, and MoMaS hard leads to a higher angle than the $\mathrm{Fe}-\mathrm{Cr}$ mineral test. Iter-ative methods result in a worse direction than direct methods, and only the Valocchi test case is solved with an accurate direction by all the iterative meth- ods. Imposing lower convergence criteria $\left(10^{-12}\right)$ on GMRES leads to a worse direction than using the usual criteria $\left(10^{-8}\right)$. Using preconditioning methods leads to a better direction when associated with a direct method (LU Equil and DGESVX), but the conclusionis less clear for the iterative GMRES Equil method. Depending on the test case, the direction can be worse (Valocchi, MoMaS easy, MoMaS medium) or better (gallic acid, pyrite, MoMaS hard, $\mathrm{Fe}-\mathrm{Cr}$ mineral)

The influence of the condition number on the angle (see Appendix 5) indicates that the direction is correct for direct methods when the condition number is less than $10^{15}$. For

iterative methods, the limit to obtain an accurate direction is a condition number less than $10^{8}$, excepted for the Gauss-

Seidel method, which produces wrong directions for low condition numbers. If the condition number increases, the behaviour of the direction becomes noisy. Since the relative error on the norm increases regularly until the condition number reaches the limit of $10^{8}$ or $10^{15}$, the angle is accu-

rately defined until this condition number limit is reached. Using preconditioned methods leads to a better direction for the LU Equil and the GMRES Equil methods when the condition number is higher than $10^{15}$ for some cases (Appendix

5 (E-21, E-23 to E-25, and E-30)) but to a worse direction for other cases (Appendix 5 (E-26 and E-29)).

We present two successful direct methods, LU and LU QUAD; one iterative method, GMRES (both tested versions, GMRES and GMRES $10^{-12}$ ); and two precondi-

tioned methods, LU Equil and the GMRES Equil. By comparing the relative error on the norm (Appendix 4 (D-21 to D-30)), the successful methods can be ranked from the lowest to highest error: LU QUAD, GMRES $10^{-12}$, GMRES

Table 4 Algorithm for equilibrium computation

\begin{tabular}{|c|c|}
\hline $\operatorname{cond}(Z)$ & Inversion method \\
\hline$>10^{30}$ & LU QUAD Equil \\
\hline \multicolumn{2}{|c|}{$10^{30} \geq \operatorname{cond}(Z)>10^{14}$ LU QUAD } \\
\hline $\begin{array}{c}14 \geq \\
\text { (2) Spring }\end{array}$ & + \\
\hline
\end{tabular}$$
+\quad \geq
$$

more complicated. For all the tests cases (Appendix 5 (E- 815 21 to E-25, E-27, E-28, and E-30)), LU QUAD gives the 816 best direction, followed by LU Equil, LU, GMRES Equil, 817 and GMRES $10^{-12}$. The MoMaS medium (Appendix 5 (E- 81826 )) and MoMaS hard (Appendix 5 (E-29)) test cases lead 819 to the same conclusion, except GMRES Equil which gives 820 the worst direction.

\subsection{Efficiency of the methods}

The speed of the methods is studied by recording the com823 putation time for each test case and plotting the mean CPU 824 time for each test case and each method (see Fig. 5). As 825 expected, the computation times are very short (less than 1 $\mathrm{ms}$ ) because the systems to solve are small.

Figure 5 shows the influence of the system size. For all methods, the computation time increases with the number of unknowns. The results show that the iterative methods are less sensitive to the system size than the direct methods. For the iterative methods, the number of iterations is important and depends on the first guess and other factors. The slowest method is LU OUAP for which a large
amount of computation time is detoted to the translation of

fipgure-precision real to guadr ple-precision real and back

the reference solution, which requires more time.

The UMF method is the slowest double-precision direct method, but its multifrontal block strategy becomes interesting for laige system. The resplution of the Morel Morgan.

the MoMaS easy test for the UMF method, whereas it takes 190 times more time for the LU method.

method. The two most robust iterative methods, BiCGStab 847 and GMRES, are rapid, sometimes more so than the direct 848 robust methods, LU and UMF, especially for large systems 849 (MorelMorgan test case). GMRES is less case-dependent 850 than BiCGStab, leading to similar computing time, regard-

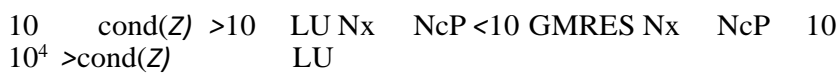
831 832 3

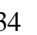

\section{.} .

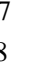


of

the

test

cas

e. 85

2

A

(LU Equil, DGESVX, and GMRES Equil) or decreasing the convergence criteria for an iterative method (GMRES

$10^{-12}$ ) leads to increased computing time. The computing time for preconditioning does not depend only on the system's size: the Valocchi, MoMaS easy, and MoMaS medium test cases (system size of $5 \times 5$ ) are solved with the same computing time for all the direct methods, but their resolution when using LU QUAD Equil, LU Equil, and GMRES Equil is faster.

Appendix 6 shows the computation time (log scale) for 861 
Fig. 6 Evolution of $\mathrm{NR}_{\text {relativeerror }}$ as a function of the Newton-Raphson iteration for the pyrite test case

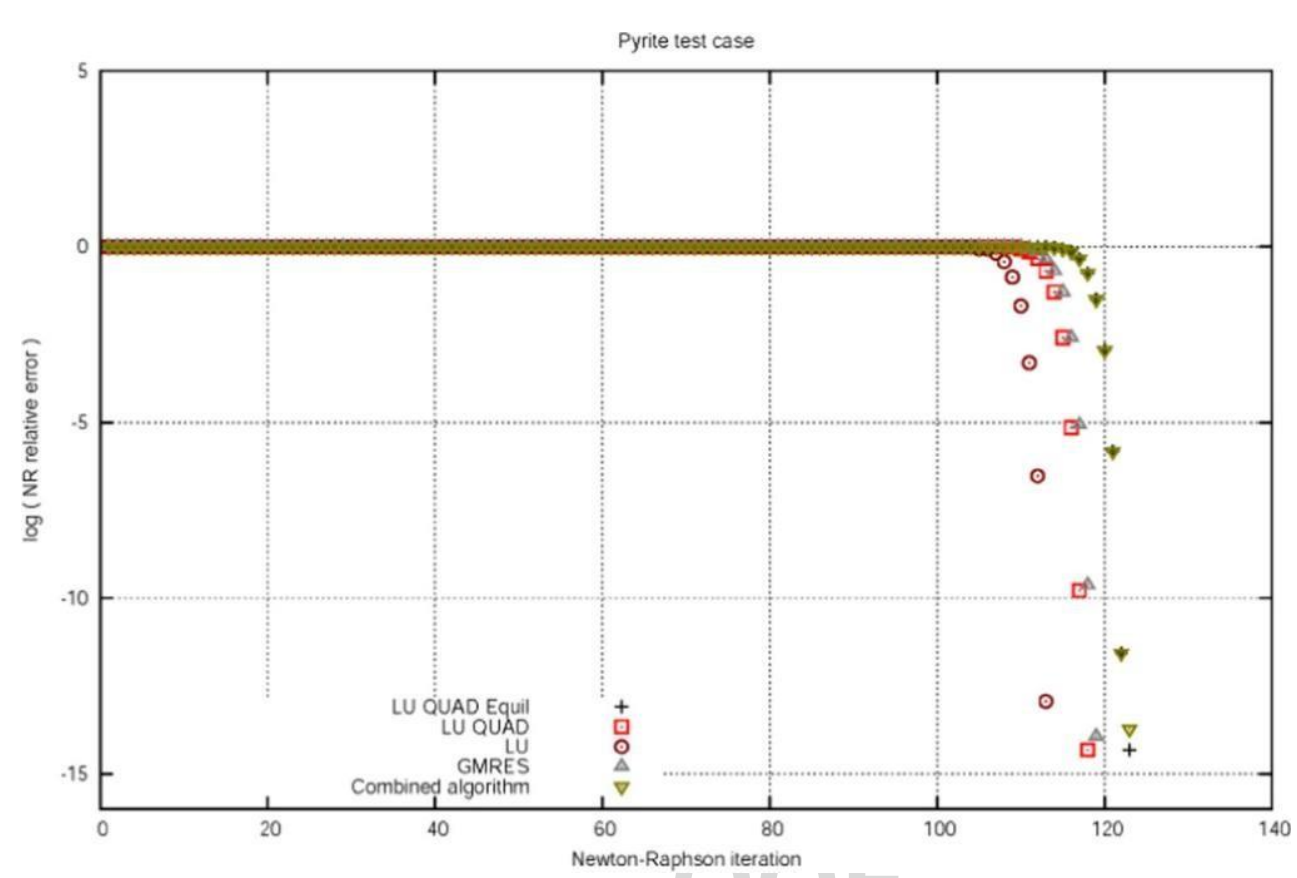

the computation time of the direct methods does not depend on the condition number of the system. The LU method is usually 10 times faster than the UMF method, except for the Morel-Morgan test case, in which LU is only 1.5 times faster.

In Appendix 6 (F-11 to F-20), the general tendency for the iterative methods is to require the same computation time, independent of the condition number. The oscillations presented by the curves seem to be not related to the condition number. For the test case without minerals, the Gauss-Seidel method is efficient. The two most robust methods, BiCGStab and GMRES, are often the third and fourth fastest methods (Gauss-Seidel and SYMMBK are the fastest).

\section{Proposal of a new algorithm}

Based on our results, we propose an algorithm to optimize the resolution of a chemical system using a NewtonRaphson-like method.

Examining the failure ratio results, seven methods are eligible: LU and LU QUAD as direct methods, GMRES and Gauss-Seidel (if no minerals) as iterative methods, LU Equil and GMRES Equil as preconditioned methods, and the reference method (LU QUAD Equil).

Because these methods are included in a Newton minimization procedure, the most important accuracy criterion is the direction of the minimization, i.e. the angle between the reference and the calculated solution. The behaviour of this direction is strongly correlated with the condition number of the system and is correct if the condition number is less than the critical value and wrong if the condition number is greater than the critical value (see Appendix 5). The critical condition number is $10^{8}$ for GMRES, $10^{16}$ for the double-precision direct methods, $10^{32}$ for LU QUAD, and case-dependent for preconditioned methods $\left(10^{20}\right.$ to $\left.10^{60}\right)$. Gauss-Seidel leads to wrong directions for very low condition numbers (Appendix 5 (E-11 and E-12)).

In terms of efficiency, the most rapid method is GaussSeidel when it is available. The second most efficient method is LU for small systems (less than $10 \times 10$ ) or GMRES for larger systems (more than $10 \times 10$ ), and the slowest method is LU QUAD. For small systems (less than $5 \times 5)$, LU Equil is as fast as GMRES but becomes slower as the system size increases.

We recommend using LU, LU QUAD, GMRES, and the reference method LU QUAD Equil. Gauss-Seidel should be rejected because of its wrong direction, and equilibration does not sufficiently improve the behaviour of doubleprecision routines.

Using Eq. 25, it is possible to estimate the condition number of the system without additional computation. This estimation enables the selection of the best-adapted method depending on the system size and condition number.

The goal is to use the most robust method (LU QUAD with preconditioning) for high condition number systems (more than $10^{32}$ ) in the first Newton-Raphson iterations. When the condition number is sufficiently decreased, the preconditioning becomes useless and LU QUAD can be used until the condition number is less than $10^{16}$. Then, a faster method is used to obtain a coarse approximation of the solution, LU for small systems and GMRES for large systems (more than $10 \times 10$ ). To find the exact solution, the LU direct method is used. 
Fig. 7 Evolution of

$\mathrm{NR}_{\text {relativeerror as a function of the }}$ Newton-Raphson iteration for the MoMaS hard case

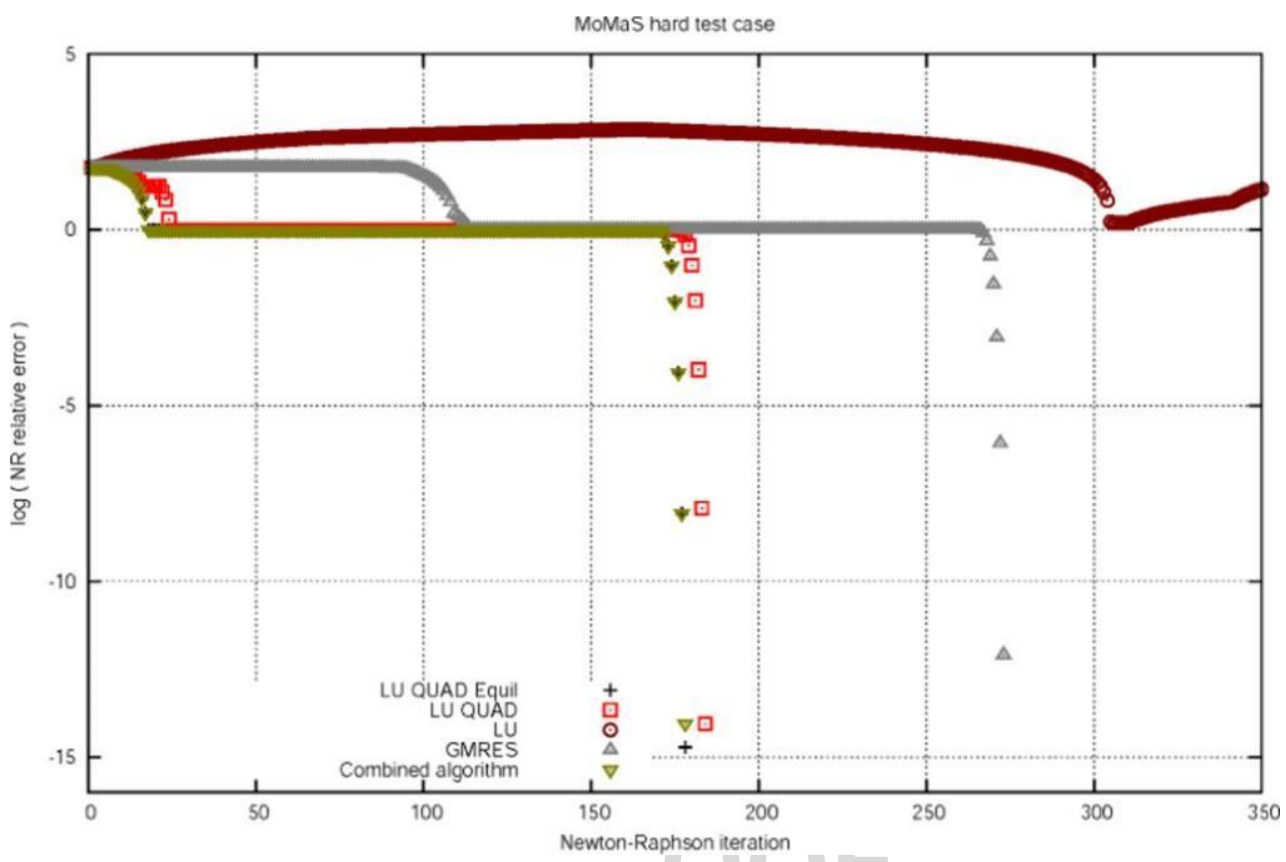

We propose the algorithm presented in Table 4 and compare it with several inversion methods in a NewtonRaphson algorithm. The 10 chemical test cases are solved using the combined algorithm or one of the selected methods: LU QUAD Equil (used as the reference solution), LU QUAD, LU, and GMRES. Appendix 7 shows the evolutio.. of the $\mathrm{NR}_{\text {re: }}$ :eerror (7) as a function of the Newton-Raphson iterations

Figure 6 shows that all the methods are equivalent for fasy test cases (see Appendix 7 (G-1 to G-3)). Nevertheless, the use of LU inversion leads to non-convergence, even if the test is easy, as observed for the Valocchi test (Appendix

Fig. 8 Computation time (s) as a function of test case and algorithm

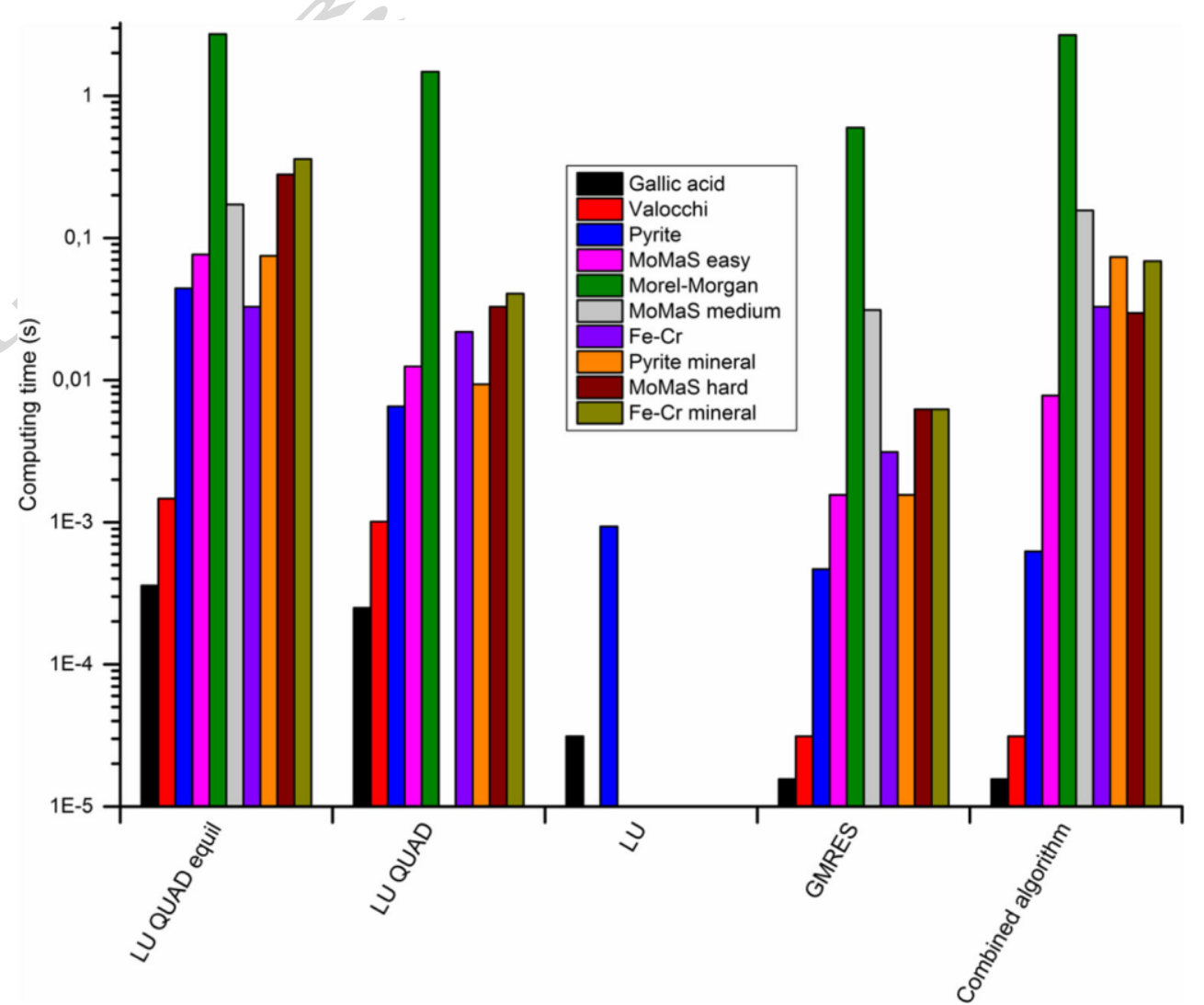


7 (G-2)). If the difficulty of the test increases, the lower accuracy of GMRES (compared to the quadruple-precision routine used in LU QUAD Equil, LU QUAD, and the combined algorithm) leads to a greater number of Newton iterations, as shown in Fig. 7 for the MoMaS hard case. This point is confirmed for other cases (see Appendix 7 (G-4 to G-9)). For the Fe-Cr mineral case (see Appendix 7 (G-10)), only LU QUAD Equil and the combined algorithm can solve the problem. Other methods lead to non-convergence, due to overflow for the GMRES algorithm (overflow appears in the Newton algorithm and is not due to GMRES itself) and because LU QUAD and LU are unable to give an accurate descent direction.

Appendix 7 (G-11) shows the evolution of the rela-tion between the norm of $Y$ and the condition number ofthe Jacobian matrix during the minimization process. This figure is similar to Fig. 1, confirming the empirical relation (25). This relation cannot be used close to the solution, and the condition number tends to be a case-dependent limit for very low $|Y| .||$

Nevertheless, the number of iterations is not the critical point. Because the time required by one iteration changes depending on the method used, we have to consider the total computation time. By plotting the total computation time required to solve each test case depending on the algorithm used (see Fig. 8), we can see that

(i) LU QUAD Equil, as expected, is the slowest. Nevertheless, this method allows the convergence of the Newton-Raphson method for all test cases.

(ii) LU QUAD is slightly faster. The difference between LU QUAD Equil and LU QUAD gives an indica- tion of the time used for matrix equilibration. This time is greater for pyrite, MoMaS easy, pyrite mineral, MoMaS hard, and Fe-Cr mineral than for the other test cases.

(iii) LU is fast when it leads to convergence, but this method results in a very weak Newton-Raphson algorithm.

(iv) GMRES always results in the fastest Newton-Raphson algorithm. It has been shown (Fig. 7, Appendix 7 (G$8)$ ) that the number of required iterations can be twice the number for other methods, but we show (Fig. 5) that the GMRES method is faster than the other methods.

(v) The proposed combined algorithm leads to intermediate computing times, equivalent to those of $\mathrm{LU}$ QUAD Equil and LU QUAD, depending on the case.

According to our results, GMRES should be systematically used because it is fast and usually leads to convergence of the Newton-Raphson algorithm. The combined algorithm should be used for very high condition numbers or for recomputing a failed run.
In this work, we focus on the resolution of small linear systems generated using the Newton-Raphson algorithm to solve equilibrium chemistry problems. For the first time, we propose a study of the condition number of such linear systems and find that the range of values covered is unusually large. This characteristic leads to specific numerical problems, with matrices that are quite small (approximately 10 $\times 10$ ) but very badly conditioned (up to $10^{100}$ ). Ten different chemical systems are studied.

There is a strong linear relationship between the logarithm of the condition number of the matrix and the logarithm of the norm of the objective function. This factor can be exploited to create efficient algorithms. This relation is strictly an empirical one and is not valuable for low condition numbers.

A wide variety of linear solvers have been tested, and several direct and iterative solvers are selected. Some of these solvers are specific for a class of matrix, symmetric or positive definite, while others are generic. A preconditioning method (matrix equilibration) has also been tested to reduce the conditioning of the systems.

According to our selected test cases, only the LU and LU QUAD direct methods, the GMRES iterative method, and LU Equil and GMRES Equil preconditioned methods are sufficiently robust to solve all the tests.

According to the size of the chemical tests, the LU method is faster than the GMRES method. However, our results for the $\mathrm{Fe}-\mathrm{Cr}$ mineral and Morel-Morgan cases show that GMRES is preferable for larger chemical systems (more than 10 components). Chemical systems with more than 10 components have not been frequently modelled in the past decade. However, the use of geochemical databases makes the construction of large geochemical systems easier, and the increase in computation capacities makes it possible. For very large geochemical systems, we recommend the GMRES method.

We also propose using the linear relationship between the condition number of the Jacobian matrix and the norm of the objective function to develop an efficient algorithm.

The classic LU method is not a good choice. Its weakness is its low robustness for challenging test cases. We recommend using the GMRES method, which is fast and usually leads to convergence of the Newton-Raphson algorithm. For very high condition numbers (more than $10^{100}$ ), we recommend the most robust LU QUAD Equil method. When the Newton-Raphson method is sufficiently near the solution to decrease the condition number, the faster GMRES method can be used. By using the linear relationship between $\operatorname{cond}(Z)$ and $\|Y\|$, the transition between the two methods can be achieved without computing the condition number (which is very expensive). 
This work explores a new research field by studying geochemical computation from a condition number point of view. We attempted to benchmark a wide variety of linear solvers, but it was not possible to explore the flexibility of all the tested solvers. This study will help us to eliminate some solvers so that our future work can focus on the most promising: LU, LU QUAD, GMRES, LU Equil, and GMRES Equil. Some points for future exploration are as follows:

(i) We did not extensively test the robustness and the efficiency of the Newton-Raphson algorithm. Further work should examine the influence of the initial Newton-Raphson guess to confirm our conclusions about the high efficiency of the GMRES method.

(ii) The accuracy of iterative methods depends on the value of the convergence criterion (which we set to $10^{-8}$ ) and on the method used to check the convergence (we used the default method). Moreover, the efficiency can vary depending on the initial guess provided by the user. In this work, we used the easiest initial guess: the residual for the tests from the Newton-Raphson method and the previous NewtonRaphson step for the test in a Newton-Raphson algorithm. We believe that it is possible to make a better choice, markedly enhancing the efficiency of the iterative methods.

(iii) The GMRES method allows the use of left and/or right preconditioners. These preconditioners can increase the robustness, accuracy, and efficiency of the method. More generally, several classes of preconditioners that may reduce the condition number of the linear system can be used $[65,66]$. In this work, we explored the use of one preconditioner: matrix equilibration. However, other classes of preconditioners may be more efficient.

(iv) Previous works have addressed the use of methods to solve geochemical equilibria other than the NewtonRaphson method [17, 44, 49, 67]. It has been shown [17] that an efficient algorithm can be obtained by combining a zero-order method with the NewtonRaphson approach.

(v) The size of the chemical tests presented here is representative of the sizes actually used in environmental studies. We have shown that the GMRES method may be efficient for large systems. In anticipation of future needs, it may be useful to test chemical systems larger than the Morel-Morgan system.

(vi) Part of the Newton minimization related to very large condition numbers (far from the solution) can be performed using random methods; GMRES is efficient even though its descent direction is not accurate for high condition numbers. Some methods, such as 1095 simulated annealing and particle swarm optimization, 1096 could be used in future research.

These factors should be explored in light of the results presented in this study. We proposed a large set of chemical tests, a criterion to determine the difficulty of these tests (the condition number), and a panel of numerical methods that should be studied preferentially.

As a more general consideration, the reader should pay particular attention to the old Morel-Morgan test case and the more realistic pyrite test case. The Morel-Morgan test uses $\mathrm{Fe}^{2+}$ and $\mathrm{Fe}^{3+}, \mathrm{Co}^{2+}$ and $\mathrm{Co}^{3+}$, and $\mathrm{SO}_{4-}^{2-}$ and $\mathrm{S}^{2-}$ as components whereas the pyrite case uses $\mathrm{O}_{2}, \mathrm{Fe}^{2+}, 1107$ and $\mathrm{SO}_{4}^{2-}$. The first studies on geochemical computation 1108 avoided redox problems. We show that redox problems 1109 lead to higher condition numbers because the stoichiomet- 1110 ric coefficients and equilibrium constants cover a wider 1111 range. Several geochemical databases avoid the introduc- 1112 tion of redox reactions. There is sometimes a good reason to

1113 not write redox reactions as equilibria (slow reaction rates, 1114 irreversible reactions) as done in Arora et al. [2]. However, 1115 the reason is sometimes numeric, and redox reactions are 1116 avoided because they lead to non-convergence.

1117

We propose the use of quadruple-precision real for chal- 1118 lenging chemical systems. In this work, the core of the 1119 geochemical code is conserved as double-precision real, 1120 and only the linear system tool is set as quadruple preci- 1121 sion. Rewriting an entire geochemical code in a quadruple- 1122 precision format will result in robust code but at the cost 1123 of an important and rebarbative work as well as efficiency. In this stage of our research, we do not recommend such an effort because implementing LU decomposition using quadruple-precision real is very efficient, requiring only a minor modification of existing code and reducing the computation time.

Acknowledgments Hela Machat has been supported by a grant from the Tunisian Government. This work is supported by the BRGMCNRS CUBICM project. We thank the reviewers for their helpful comments.

Compliance with Ethical Standards Compliance with ethical standards

Conflict of interests The authors declare that they have no conflict of interest.

Ethical approval This article does not contain any studies with 1138 human participants or animals performed by any of the authors. 
Comput Geosci

Q11142

Appendix 1

Morel's table of chemical test cases

1144

\begin{tabular}{|c|c|c|c|c|c|c|c|c|}
\hline & & & & & & & & \\
\hline & & $\mathrm{X} 1$ & $\mathrm{X} 2$ & $\mathrm{X} 3$ & $\mathrm{X} 4$ & $\mathrm{X} 5$ & $S$ & $\log (\mathrm{K})$ \\
\hline 1 & $\mathrm{X} 1$ & 1 & 0 & 0 & 0 & 0 & 0 & 0.00 \\
\hline 2 & $\mathrm{X} 2$ & 0 & 1 & 0 & 0 & 0 & 0 & 0.00 \\
\hline 3 & $\mathrm{X} 3$ & 0 & 0 & 1 & 0 & 0 & 0 & 0.00 \\
\hline 4 & $\mathrm{X} 4$ & 0 & 0 & 0 & 1 & 0 & 0 & 0.00 \\
\hline 5 & $\mathrm{X} 5$ & 0 & 0 & 0 & 0 & 1 & 0 & 0.00 \\
\hline 6 & $\mathrm{C} 1$ & 0 & -1 & 0 & 0 & 0 & 0 & -12.00 \\
\hline 7 & $\mathrm{C} 2$ & 0 & 1 & 1 & 0 & 0 & 0 & 0.00 \\
\hline 8 & $\mathrm{C} 3$ & 0 & -1 & 0 & 1 & 0 & 0 & 0.00 \\
\hline 9 & $\mathrm{C} 4$ & 0 & -4 & 1 & 3 & 0 & 0 & -1.00 \\
\hline 10 & $\mathrm{C} 5$ & 0 & 4 & 3 & 1 & 0 & 0 & 35.00 \\
\hline 11 & C6 & 0 & 10 & 3 & 0 & 0 & 0 & 32.00 \\
\hline 12 & $\mathrm{C} 7$ & 0 & -8 & 0 & 2 & 0 & 0 & -4.00 \\
\hline 13 & S & 0 & 0 & 0 & 0 & 0 & 1 & 0.00 \\
\hline 14 & CS1 & 0 & 3 & 1 & 0 & 0 & 1 & 6.00 \\
\hline 15 & $\mathrm{CS} 2$ & 0 & -3 & 0 & 1 & 0 & 2 & -1.00 \\
\hline 16 & CP1 mineral & 0 & 1 & 3 & 0 & 0 & 0 & 10.90 \\
\hline \multirow[t]{7}{*}{17} & $\mathrm{CP} 2$ mineral & 0 & 1 & 0 & 0 & 1 & 0 & 1.30 \\
\hline & Total (M) & 0.3 & 0.3 & 0.3 & 2 & 0.3 & 10 & \\
\hline & $X$ value & 0.1 & Variable & Variable & Variable & $1.00 E-03$ & $1.00 E-03$ & \\
\hline & Min value & & $1.00 E-1$ & $1.00 E-1$ & $1.00 E-1$ & & & \\
\hline & & & 5 & 5 & 5 & & & \\
\hline & Max value & & $1.00 E-02$ & $1.00 E-02$ & $1.00 E-02$ & & & \\
\hline & $X$ initial value & 0.1 & $1.00 E-07$ & $1.00 E-07$ & $1.00 E-03$ & $1.00 E-03$ & $1.00 E-03$ & \\
\hline
\end{tabular}

1146

\section{Appendix 2}

Jacobian matrix properties

B-1 Ratio of diagonal dominant matrices as a function of the condition number for the 10 chemical test cases

B-2 Ratio of positive definite matrices as a function of the condition number for the 10 chemical test cases

\section{Appendix 3}

Evolution of the failure ratio of the 16 selected methods as a function of the condition number for the 10 chemical test cases

C-1-C-7: Direct methods, chemical cases without minerals

C-8-C-10: Direct methods, chemical cases with minerals

C-11-C-17: Iterative methods, chemical cases without minerals

C-18-C-20: Iterative methods, chemical cases with himerinas
C-21-C-27: Preconditioned methods, chemical cases without minerals

C-28-C-30: Preconditioned methods, chemical cases with minerals

1162

1163

1164

1165

\section{Appendix 4}

1166

Evolution for the relative residual on norm of the 16 selected methods as a function of the condition number for the 10 chemical test cases

D-1-D-7: Direct methods, chemical cases without minerals

D-8-D-10: Direct methods, chemical cases with minerals

D-11-D-17: Iterative methods, chemical cases without minerals

D-18-D-20: Iterative methods, chemical cases with minerals

D-31-D-37: Preconditioned methods, chemical cases without minerals

D-38-D-40: Preconditioned methods, chemical cases with minerals 


\section{Appendix 5}

Evolution for the angle between the reference and computed solution of the 16 selected methods as a function of the condition number for the 10 chemical test cases

E-1-E-7: Direct methods, chemical cases without minerals

E-8-E-10: Direct methods, chemical cases with minerals

E-11-E-17: Iterative methods, chemical cases without minerals

E-18-E-20: Iterative methods, chemical cases with minerals

E-21-E-27: Preconditioned methods, chemical cases without minerals

E-28-E-30: Preconditioned methods, chemical cases with minerals

\section{Appendix 6}

Evolution for the computation time of the 16 selected methods as a function of the condition number for the 10 chemical test cases

F-1-F-7: Direct methods, chemical cases without minerals

F-8-F-10: Direct methods, chemical cases with minerals

F-11-F-17: Iterative methods, chemical cases without minerals

F-18-F-20: Iterative methods, chemical cases with minerals

F-21-F-27: Preconditioned methods, chemical cases without minerals

F-28-F-30: Preconditioned methods, chemical cases with minerals

\section{Appendix 7}

Evolution of the Newton-Raphson residual as a function of the number of iterations for the 10 chemical test cases and the 5 tested algorithms

G-1-G-7: Chemical test without mineral

G-8-G-10: Chemical test with minerals

G-11: Evolution of the relation between $Y\|\|$ and the condition number of the Jacobian matrix $\mathrm{Z}$ during minimization

\section{References}

1. Walter, A.L. et al.: Modeling of multicomponent reactive transport in groundwater. 2. Metal mobility in aquifers impacted by acidic mine tailings discharge. Water Resour. Res. 30(11), 3149-3158 (1994)
2. Arora, B. et al.: A reactive transport benchmark on heavy metal 1221 cycling in lake sediments Computational Geosciences (2014) 1222

3. De Windt, L., Leclercq, S., Van der Lee, J.: Assessing the durabil- 1223 ity of nuclear glass with respect to silica controlling processes in 1224 a clayey underground disposal. In: 29th International Symposium 1225 on the Scientific Basis for Nuclear Waste Management XXIX. 1226 Materials Research Society Symposium Proceedings, Ghent; Bel- 1227 gium (2005)

4. Hoteit, H., Ackerer, P., Mose, R.: Nuclear waste disposal simula- 1229 tions: Couplex test cases. Comput. Geosci. 8(2), 99-124 (2004) 1230

5. Tompson, A.F.B., et al.: On the evaluation of groundwater contam- 1231 ination from underground nuclear tests. Environ. Geol. 42(2-3), 1232 235-247 (2002)

6. Andre, L., et al.: Numerical modeling of fluid-rock chemical 1234 interactions at the supercritical CO2-liquid interface during CO2 1235 injection into a carbonate reservoir, the Dogger aquifer (Paris 1236 Basin, France). Energy Convers. Manag. 48(6), 1782-1797 (2007) 1237

7. Kang, Q., et al.: Pore scale modeling of reactive transport involved 1238 in geologic CO2 sequestration. Transp. Porous Media 82(1), 197- 1239 213 (2010)

8. Navarre-Sitchler, A.K., et al.: Elucidating geochemical response of shallow heterogeneous aquifers to $\mathrm{CO} 2$ leakage using highperformance computing: implications for monitoring of $\mathrm{CO} 2$ sequestration. Adv. Water Resour. 53(0), 45-55 (2013)

9. Pruess, K. et al.: Code intercomparison builds confidence in numerical simulation models for geologic disposal of $\mathrm{CO}$. Energy 29(9-10), 1431-1444 (2004)

10. Regnault, O., et al.: Etude experimentale de la reactivite du CO2 supercritique vis-a-vis de phases minerales pures. Implications pour la sequestration geologique de CO2. Compt. Rendus Geosci. 337(15), 1331-1339 (2005)

11. Valocchi, A.J., Street, R.L., Roberts, P.V.: Transport of ion- 1252 exchanging solutes in groundwater: chromatographic theory and 1253 field simulation. Water Resour. Res. 17, 1517-1527 (1981) 1254

12. Lichtner, P.C.: Continuum model for simultaneous chemical reac- 1255 tions and mass transport in hydrothermal systems. Geochim. 1256 Cosmochim. Acta 49(3), 779-800 (1985) 1257

13. Appelo, C.A.J.: Hydrogeochemical transport modelling. Proceed. 1258 Inf.-Comm. Hydrol. Res. TNO 43, 81-104 (1990)

14. Yeh, G.T., Tripathi, V.S.: A critical evaluation of recent develop- 1260 ments in hydrogeochemical transport models of reactive multi- 1261 chemical components. Water Resour. Res. 25, 93-108 (1989) 1262

15. Carrayrou, J. et al.: Comparison of numerical methods for sim- 1263 ulating strongly nonlinear and heterogeneous reactive transport 1264 problems - the MoMaS benchmark case. Computational Geo- 1265 sciences 14(3), 483-502 (2010)

16. Hammond, G.E., Valocchi, A.J., Lichtner, P.C.: Modeling mul- 1267 ticomponent reactive transport on parallel computers using 1268 Jacobian-Free Newton Krylov with operator-split preconditioning. 1269In: Hassanizadeh, S.M. (ed.) Developments in water science, com- 1270 putational methods in water resources, Proceedings of the XIVth 1271 International Conference on Computational Methods in Water 1272 Resources (CMWR XIV), pp. 727-734. Elsevier (2002) 1273

17. Carrayrou, J., Mosé, R., Behra, P.: New efficient algorithm for 1274 solving thermodynamic chemistry. AIChE J. 48(4), 894-904 1275 (2002)

18. Amir, L., Kern, M.: A global method for coupling transport with 1277 chemistry in heterogeneous porous media. Comput. Geosci. 14(3), 1278 465-481 (2010)

19. Quarteroni, A., Sacco, R., Saleri, F.: Numerical mathematics. In: 1280 Marsden, J.E., Sirovich, L., Antman. S.S. (eds.) Texts in Applied 1281 Mathematics. 2nd edn. Springer, Heidelberg (2007) 1282

20. Axelsson, O., et al.: Direct solution and incomplete factoriza- 1283 tion preconditioned conjugate gradient methods. Comparison of 1284 
algebraic solution methods on a set of benchmark problems in linear elasticity, in STW report. 2000, Department of Mathematics, Catholic University of Nijmegen: Nijmegen, The Netherlands. pp. 1-36

21. Barrett, R., Berry, M., Chan, T.F., Demmel, J., Donato, J., Dongarra, J., Eijkhout, V., Pozo, R., Romine, C., Van Der Vorst, H. Templates for the solution of linear systems: building blocks for iterative methods, 2nd edn. SIAM, Philadelphia (1994)

22. Gould, N.I.M., Hu, Y., Scott, J.A.: A numerical evaluation of sparse direct solvers for the solution of large sparse, symmetric linear systems of equations. 2005, Council for the Central Laboratory of the Research Councils

23. Allaire, G., Kaber, S.M. In: Marsden, J.E., Sirovich, L., Antman, S.S. (eds.): Numerical linear algebra. Texts in applied mathematics. Springer, New York (2008)

24. Baldwin, C., et al.: Iterative linear solvers in a $2 \mathrm{D}$ radiationhydrodynamics code: methods and performance. J. Comput. Phys. 154(1), 1-40 (1999)

25. Chao B.T., L.H.L., Scott, E.J.: On the solution of ill-conditioned, simultaneous, linear, algebraic equations by machine computation, in University of Illinois Bulletin. 1961, University of Illinois

26. Hadjidimos, A.: Successive overrelaxation (SOR) and related methods. J. Comput. Appl. Math. 123(1-2), 177-199 (2000)

27. Kalambi, I.B.: A comparison of three iterative methods for the solution of linear equations. J. Appl. Sci. Environ. Manag. 12(4), 53-55 (2008)

28. Klisinski, M., Runesson, K.: Improved symmetric and nonsymmetric solvers for FE calculations. Adv. Eng. Softw. 18(1), 41-51 (1993)

29. Schenk, O., Gartner, K.: Solving unsymmetric sparse systems of linear equations with PARDISO. Fut. Gener. Comput. Syst. 20(3), 475-487 (2004)

30. Xue, X.J. et al.: A direct algorithm for solving ill-conditioned linear algebraic systems. JCPDS-Int. Centre Diffract. Data Adv. X-ray Anal. 42, 629-633 (2000)

31. Pyzara, A., Bylina, B., Bylina, J.: The influence of a matrix condition number on iterative methods' convergence (2011)

32. Hoffmann, J., Kräsutle, S., Knabner, P.: A parallel global-implicit 2-D solver for reactive transport problems in porous media based on a reduction scheme and its application to the MoMaS benchmark problem. Comput. Geosci. 14(3), 421-433 (2010)

33. Soleymani, F.: A new method for solving ill-conditioned linear systems. Opuscula Math. 33(2), 337-344 (2013)

34. Morel, F., Morgan, J.: A numerical method for computing equilibria in aqueous chemical systems. Environ. Sci. Technol. 6(1), 58-67 (1972)

35. Morel, F.M.M.: Principles of aquatic chemistry. Wiley Interscience, New York (1983)

36. De Windt, L. et al.: Intercomparison of reactive transport models applied to UO2 oxidative dissolution and uranium migration. J. Contam. Hydrol. 61(1-4), 303-312 (2003)

37. Jauzein, M. et al.: A flexible computer code for modelling transport in porous media: impact. Geoderma 44(2-3), 95113 (1989)

38. Parkhurst, D.L., Appelo, C.A.J.: User's guide to PHREEQC (version 2) - a computer program for speciation, batch-reaction, one-dimensional transport, and inverse geochemical calculations. Water Resour. Invest., Editor. 1999: Denver. p. 312

39. Van der Lee, J.: CHESS another speciation and surface complexation computer code. E.d.M.d. Paris, Editor. 1993: Fontainebleau. p. 85

40. Westall, J.C.: MICROQL: a chemical equilibrium program in BASIC. Computation of adsorption equilibria in BASIC. S.F.I.o.T. EAWAG, Editor. 1979: Dübandorf. p. 42
41. Westall, J.C.: FITEQL ver. 2.1. 1982: Corvallis

42. Westall, J.C., Zachary, J.L., Morel, F.M.M.: MINEQL: a computer program for the calculation of chemical equilibrium composition of aqueous system. R.M.P. Laboratory, Editor. 1976: Cambridge. p. 91

43. Walter, L.J., Wolery, T.J.: A monotone-sequences algorithm and FORTRAN IV program for calculation of equilibrium distributions of chemical species. Comput. Geosci. 1, 57-63 (1975)

44. Wigley, T.M.L.: WATSPEC: a computer program for determining the equilibrium speciation of aqueous solutions. B.G.R.G. Tech. Bull., Editor. 1977. p. 49

45. Jennings, A.A., Kirkner, D.J., Theis, T.L.: Multicomponent equilibrium chemistry in groundwater quality models. Water Resour. Res. 18, 1089-1096 (1982)

46. Cederberg, A., Street, R.L., Leckie, J.O.: A groundwater mass transport and equilibrium chemistry model for multicomponent systems. Water Resour Res. 21, 1095-1104 (1985)

47. Yeh, G.T., Tripathi, V.S.: A model for simulating transport of reac- 1366tive multispecies components: model development and demonstra- 1367 tion. Water Resour. Res. 27(12), 3075-3094 (1991)

48. Carrayrou, J.: Looking for some reference solutions for the reactive transport benchmark of MoMaS with SPECY. Comput. Geosci. 14(3), 393-403 (2010)

49. Brassard, P., Bodurtha, P.: A feasible set for chemical speciation problems. Comput. Geosci. 26(3), 277-291 (2000)

50. Carrayrou, J., Kern, M., Knabner, P.: Reactive transport benchmark of MoMaS. Comput. Geosci. 14(3), 385-392 (2010)

51. Fendorf, S.E., Li, G.: Kinetics of chromate reduction by ferrous iron. Environ. Sci. Technol. 30(5), 1614-1617 (1996)

52. Chilakapati, A. et al.: Groundwater flow, multicomponent transport and biogeochemistry: development and application of a coupled process model. J. Contam. Hydrol. 43(3-4), 303-325 (2000)

53. Knight, P., Ruiz, D., Ucar, B.: A symmetry preserving algorithm for matrix scaling. SIAM J. Matrix Anal. Appl. 35(3), 931-955 (2014)

54. Golub, H.V., Van Loan, C.F.: Matrix computations. 3rd ed. The Johns Hopkins University Press, Baltimore (1996)

55. Davis, T.A., Duff, I.S.: A combined unifrontal/multifrontal method for unsymmetric sparse matrices. ACM Trans. Math. Softw. 25(1), 1-20 (1999)

56. Woźnicki, Z.: On performance of SOR method for solving nonsymmetric linear systems. J. Comput. Appl. Math. 137(1), 145176 (2001)

57. Saad, Y., Van Der Vorst, H.A.: Iterative solution of linear systems in the 20th century. J. Comput. Appl. Math. 123(1-2), 1-33 (2000)

1368

1369

1370

1371

1372

1373

1374

1375

1376

1377

1378

1379

1380

1381

1382

1383

1384

1385

1386

1387

1388

1389

1390

1391

1392

1393

1394

58. Diersch, H.J.G.: FEFLOW reference manual. DHI-WASY GmbH, Berlin (2009)

59. Van der Lee, J., et al.: Presentation and application of the reactive transport code HYTEC. In: Hassanizadeh, S.M. (ed.) Developments in Water Science, Computational Methods in Water Resources, Proceedings of the XIVth International Conference on Computational Methods in Water Resources (CMWR XIV), pp. 599-606. Elsevier (2002)

60. Press, W.H., S.A.T., Vettering, W.T., Flannery, B.P. Numerical recipes in FORTRAN: the art of scientific computation, 2nd edn., pp. 123-124. Cambridge University Press, New Yor (1992)

61. The Linear Algebra Package (LAPACK) can be obtained free of charge from the address listed here: http://www.netlib.org/lapack

62. Kincaid, D., Cheney, W. Numerical analysis: mathematics of scientific computing, 3rd edn. American Mathematical Society (2002)

63. HSL: A collection of Fortran codes for large scale scientific computation. http://www.hsl.rl.ac.uk (2013)
1395

1396

1397

1398

1399

1400

1401

1402

1403

1404

1405

1406

1407

1408

1409

1410

1411

1412 
1413

1414

1415

1416

1417
64. Chapter 8 Systems of nonlinear equations. In: Studies in computational mathematics, Claude, B. Editor. 1997, Elsevier. pp. 287-336

65. Soleymani, F.: A rapid numerical algorithm to compute matrix inversion. Int. J. Math. Math. Sci. 2012 (2012)
66. Soleymani, F.: On a fast iterative method for approximate inverse 1418 of matrices. Commun. Korean Math. Soc. 28(2), 407-418 (2013)

67. Morin, K.A.: Simplified explanations and examples of comput- 1420 erized methods for calculating chemical equilibrium in water. 1421 Comput. Geosci. 11, 409-416 (1985) 


\section{AUTHOR QUERIES}

\section{AUTHOR PLEASE ANSWER ALL QUERIES:}

Q1. Please check the provided city (Jendouba) and country (Tunisia) names in affiliation 2 if correct.

Q2. Please check if the abbreviation "LAPACK" is defined correctly. Otherwise, please provide the correct expansion.

Q3. Please check all equation citations if correctly captured or presented.

Q4. The section citation "Properties of the Jacobian matrix" found in the sentence starting "This formulation has some weaknesses" was changed to "Section 3.1" as per numbering style. Please check if correct.

Q5. As per instruction, if there are more than one appendix, they should be designated with numbers $1,2,3$, etc. With this regard, the appendices were renumbered accordingly and their citations were modified. Please advise if the labels designated for figures found in appendices $B$ to $G$ (as originally labelled) should also be modified accordingly to ensure consistency of presentation.

Q6. Missing citation for Table 1 was inserted in this sentence. Please check if appropriate. Otherwise, please provide the location of where to insert the citation/s in the main body of the text. Note that the order of main citations of tables in the text must be sequential.

Q7. Please check the captured caption of Figure 4 if correct.

Q8. Missing citation for Figure 4 was inserted in this sentence. Please check if appropriate. Otherwise, please provide the location of where to insert the citation/s in the main body of the text. Note that the order of main citations of figures in the text must be sequential.

Q9. The appendix citations found in the last two paragraphs before the "Proposal of a new algorithm" section were all changed to "Appendix 6" so that appendix citations are in sequential order. Also, the discussion in last two paragraphs fits to the description of Appendix 6. Please check if correct and amend if necessary.

Q10. Please confirm if the "Informed consent" statement is indeed applicable to this article and should be retained.

Q11. Please check if the content of Appendices 1 to 7 is correctly captured or presented.

Q12. Please check if the labels "D-31 to D-40" are indeed correct in the artwork of Appendix 4, and not "D-21 to D-30", respectively. 